\title{
MINIMAL MODELS AND DEGENERATIONS OF SURFACES WITH KODAIRA NUMBER ZERO
}

\author{
BRUCE CRAUDER AND DAVID R. MORRISON
}

\begin{abstract}
For Ueno's degenerations of abelian surfaces and for analytic flowerpot degenerations of surfaces with Kodaira number zero, we find analytically $\mathbb{Q}$-factorial terminal minimal models. All contractions, singularities and flips are given by explicit geometric constructions.
\end{abstract}

Over thirty years ago, Kodaira [Ko] gave an analysis of compact complex surfaces with a one-parameter family of elliptic curves which has served as a guide in almost all subsequent attempts to classify algebraic and complex analytic varieties. The first step in Kodaira's program was to pass to the (relative) minimal model of the surface, since Grauert $[G]$ had demonstrated that the Castelnuovo-Enriques contraction criterion was valid in the analytic category. Kodaira then analyzed all possible singular fibers which can occur on a relative minimal model. This provides a precise geometric description of such surfaces. Kodaira's analysis was extended to the number-theoretic case by Néron [N], where it has had important ramifications in the study of the arithmetic of elliptic curves.

The birational part of Kodaira's analysis was based on the well-understood birational geometry of algebraic surfaces. At the time, birational geometry in higher dimensions was quite mysterious. But the last several years have seen great advances in understanding the birational geometry of algebraic threefolds, thanks to work of Mori, Reid, Kawamata, Shokurov, Kollár, and others. Their theory selects a distinguished class of birational models of threefolds, called minimal models by analogy with the surface case, which are characterized by the property that the canonical divisor on a minimal model $X$ has nonnegative intersection number with any curve on $X$. In order to obtain these minimal models, singularities of a certain mild type (called $\mathbb{Q}$-factorial terminal singularities) must be allowed. In this class of varieties, the minimal model theorem states that beginning from a nonsingular variety $Y$, there is a sequence of birational operations which produces a variety $X$-itself either a minimal model or a variety whose canonical bundle is "negative" in a certain sense. The birational operations involved are either contractions of a divisor to a point or a curve, or "flips" of curves. Flips are a new phenomenon in dimension three: a flip is a birational map from a variety $Y$ to a variety $Y^{+}$which induces an

Received by the editors December 15, 1991.

1991 Mathematics Subject Classification. Primary 14E30, 14B15; Secondary 14E35, 14E05, $14 \mathrm{~J} 15,14 \mathrm{~K} 07$.

Research partially supported by the National Science Foundation. 
isomorphism from the complement of a curve $C$ on $Y$ to the complement of a curve $C^{+}$on $Y^{+}$(and satisfies a few other properties as well).

There are two technical difficulties inherent in applying the minimal model theorem to particular cases. One is the enormous potential complexity of terminal singularities: although they have all been listed and classified (see [R3], for example), the description is quite complicated. The second is the global nature of the algorithm specified in the minimal model theorem: one cannot decide whether a given divisor or curve is to be contracted or flipped based on purely local information about the divisor or curve.

Applying the minimal model theorem to the case of threefolds with Kodaira number one, it follows that there exist (relative) minimal models for one-parameter families of surfaces with Kodaira number zero. But due to the technical difficulties mentioned above, this minimal model is not so easy to find in particular cases.

In the case of a family of surfaces with Kodaira number zero which is birational to a semistable degeneration, the possible singular fibers are well understood, thanks to work of Kulikov [Ku], Persson-Pinkham [PP], the second author [Mrr1], and Tsuchihashi [Tsch]. In fact, many of the new phenomena in three-dimensional birational geometry were first discovered in these special cases, and the existence of minimal models for semistable degenerations of (arbitrary) surfaces was proved (prior to Mori's proof [Mr] of the general theorem) by Kawamata [Ka], Mori (unpublished), Shokurov (cf. [S]), and Tsunoda [Tsn], [My], working independently. These proofs included explicit geometric descriptions of both the terminal singularities and the flips involved in finding the minimal models.

In this paper, we will examine two additional cases of degenerations of surfaces which have been previously studied in the literature, and find the minimal models of these families in an explicit geometric way. (Our minimal models are constructed in the analytic category, and are constructed locally-by this means, we avoid the complications arising from the global nature of algebraic minimal models.) Since the previous studies in question ([U1, U2, and CM1]) were concerned with the second part of Kodaira's program (explicitly identifying possible singular fibers), as an immediate consequence we obtain complete lists of possible singular fibers in minimal models of degenerations (under certain hypotheses). Our methods include an explicit geometric description of both the terminal singularities and the flips that we use. We hope that these downto-earth descriptions will prove useful in other contexts. One application to number theory is given in [JM].

The degenerations we focus on are those with "potentially good reduction". This means that after a finite ramified cover of the parameter curve, the pulledback degeneration is birational to one with nonsingular total space and nonsingular central fiber. We study this situation in two cases: degenerations of principally polarized abelian surfaces (restricting our attention to the cases earlier treated by Ueno [U1, U2]), and degenerations which have a model which is "triple-point-free". We had earlier studied such triple-point-free degenerations and given a rough classification of central fibers [CM1]; here, we concentrate on the natural sub-class (the "flower-pot degenerations") which includes all (triplepoint-free) cases with potentially good reduction. For Ueno's degenerations, Theorem 1.1 describes the singularities, flips and birational operations needed 
to pass to the minimal models. For flower-pot degenerations, Theorem 2.7 contains the main result, including a complete list of singularities, flips and birational operations.

In $\S 1$, we describe Ueno's degeneration of abelian surfaces and how to pass to minimal models by contractions and the very simplest type of flip (Theorem 1.1). We recall known facts on flower-pot degenerations of surfaces with Kodaira number zero in $\S 2$, state our main theorem (2.7) and outline its proof. The types of singularities and contractions needed for flower-pot degenerations are established in $\S 3$, while $\S 4$ sets up the needed types of flips and a contraction criterion for minimal ruled surfaces in threefolds with singularities of the types from $\S 2$. In $\S 5$, we show how to pass to minimal models for flower-pot degenerations, using the contractions and flips from $\S \S 3$ and 4. Appendix I corrects Ueno's classification in two cases.

\section{Prerequisites}

0.1. Notation. A threefold $X$ has a cyclic quotient singularity at $P$ of type $\frac{1}{r}\left(a_{1}, a_{2}, a_{3}\right)$ if $(X, P)$ is locally analytically isomorphic to the quotient of $\mathbb{C}^{3}$ by the action of

$$
\left(z_{1}, z_{2}, z_{3}\right) \mapsto\left(\zeta^{a_{1}} z_{1}, \zeta^{a_{2}} z_{2}, \zeta^{a_{3}} z_{3}\right)
$$

where $\zeta$ is a primitive $r$ th root of unity.

0.2. Terminal Lemma [R3]. A singularity of type $\frac{1}{r}\left(a_{1}, a_{2}, a_{3}\right)$ is terminal (resp. canonical) if and only if

$$
\overline{k a_{1}}+\overline{k a_{2}}+\overline{k a_{3}}>r
$$

(resp. $\geq$ ) for all $k=1, \ldots, r-1$, where - denotes smallest residue $\bmod r$.

0.3. Contraction and simple flip. Let $X$ be a smooth threefold containing a rational curve $C$ meeting a surface $V \cong \mathbb{P}^{2}$ normally in a single point. Assume that $N_{C / X} \cong \mathscr{O}_{C}(-1) \oplus \mathscr{O}_{C}(-1)$ and $N_{V / X} \cong \mathscr{O}_{V}(-2)$. As is well known, $C$ can be blown up and the resulting exceptional $\mathbb{P}^{1} \times \mathbb{P}^{1}$ smoothly contracted "the other way". The effect of this flop on $V$ is to make $V$ into an $\mathbb{F}_{1}$ smoothly contractible to a rational curve $C^{\prime}$. Let $\varphi$ be the composition of this flop followed by the contraction, so $\varphi$ is a birational map between smooth threefolds which maps the union of $C$ and $V$ to $C^{\prime}$ and is an isomorphism elsewhere.

Note that $\varphi$ may be factored as a contraction followed by a flip as follows: Let $\sigma: X \rightarrow \bar{X}$ be the contraction of $V$ to a terminal point of type $\frac{1}{2}(1,1,1)$ and $\bar{C}$ be the image of $C$. The birational map $\bar{\varphi}=\varphi \circ \sigma^{-1}: \bar{X} \rightarrow X^{\prime}$ maps $\bar{C}$ to $C^{\prime}$ and is an isomorphism off the smooth rational curves $\bar{C}$ and $C^{\prime}$; moreover, $K_{\bar{X}} \cdot \bar{C}=-\frac{1}{2}$ while $K_{X^{\prime}} \cdot C^{\prime}=+1$. Thus $\varphi=\bar{\varphi} \circ \sigma$ factors $\varphi$ as the composition of a contraction followed by a flip. The flip $\bar{\varphi}$ will be referred to as a simple flip and is frequently used in $\S 1$ (always paired with a contraction $\sigma)$.

0.4. Notation. $S$ is a Moishezon surface if $S$ is a compact complex surface for which $\operatorname{tr} \operatorname{deg}_{\mathbb{C}} \mathbb{C}(S)=2$; equivalently, there is a desingularization $\widetilde{S} \rightarrow S$ for which $\widetilde{S}$ is projective.

0.5. Extremal Curve Lemma. If $S$ is a Moishezon surface for which $K_{S}$ is not numerically effective and $\rho(S)>2$, then there is an irreducible curve $C$ on $S$ 
with $C^{2}<0$ such that $\tilde{C}$ is an exceptional curve on $\tilde{S}$, where $\tilde{C}$ is the proper transform of $C$ under $\widetilde{S} \rightarrow S$, a desingularization of $S$.

0.6. Self-intersections on singular surfaces. Let $S$ be a Moishezon surface with isolated singular points $P_{1}, \ldots, P_{n}$ where $\left(S, P_{i}\right)$ is of type $\frac{1}{m_{i}}(1,1)$, i.e. $P_{i}$ can be resolved by a smooth rational curve of self-intersection $-m_{i}$. If $\sigma: \widetilde{S} \rightarrow S$ is the minimal resolution of $S, C$ is a curve on $S$ and $\widetilde{C}$ is its proper transform on $\widetilde{S}$, then

$$
\left(C^{2}\right)_{S}=\left(\widetilde{C}^{2}\right)_{\widetilde{S}}+\sum_{i=1}^{n} \frac{1}{m_{i}}\left(\widetilde{C} \cdot E_{i}\right)
$$

where $E_{i}=\sigma^{-1}\left(P_{i}\right)$. This follows from the fact that $\sigma^{*} C \equiv \widetilde{C}+$ $\sum_{i=1}^{n} \frac{1}{m_{i}}\left(\widetilde{C} \cdot E_{i}\right) E_{i}$, which is derived from $\left(E_{i}^{2}\right)_{\widetilde{s}}=-m_{i}$.

0.7. Cones. Let $\mathbb{F}_{n}$ be the minimal rational ruled surface having a section $\sigma_{-}$ of self-intersection $-n . \sigma_{+}$denotes a section on $\mathbb{F}_{n}$ of self-intersection $n$, and $\beta$ denotes a smooth elliptic bisection. By $\overline{\mathbb{F}}_{n}$, we mean the cone obtained by contracting $\sigma_{-}$to a point. The image of $\sigma_{-}$is called the vertex of the cone if $n \geq 2$. (In the case $\overline{\mathbb{F}}_{1} \cong \mathbb{P}^{2}$, any point can be considered to be the "vertex.")

We call the image in the cone of a fiber from the ruled surface a generator of the cone. If $F$ is a generator of a cone, then it is easy to compute that $F^{2}=\frac{1}{n}$, where $-n$ is the self-intersection of the exceptional curve in the blowup of the vertex of the cone. There are also canonical bundle formulas for the cone $V: K_{V} \cdot F=-1+(2 g-2) / n$ where $g$ is the genus of the curve over which the cone was taken, and $K_{\widetilde{V}}$ is numerically equivalent to $\pi^{*}\left(K_{V}\right)+$ $(-1+(2-2 g) / d) \sigma_{-}$, where $\pi: \widetilde{V} \rightarrow V$ is the blowup of the vertex $P$, and $\sigma_{-}=\pi^{-1}(P)$.

0.8 . Linear and numerical equivalence. We use $\sim$ to indicate linear equivalence (over $\mathbb{Z})$ and $\equiv$ to indicate numerical equivalence over $\mathbb{Q}$.

\section{UENO'S DEGENERATIONS OF ABELIAN SURFACES AND THEIR MINIMAL MODELS}

In [U1 and U2], Ueno considers projective degenerations of principally polarized abelian surfaces which are birational to smooth degenerations after a finite base-change. For $\pi: \mathscr{A} \rightarrow \Delta$ such a degeneration $\mathscr{A}$ is a nonsingular threefold, $\Delta$ is the unit disk about 0 in $\mathbb{C}$ and $\mathscr{A}_{t}$ is a nonsingular principally polarized abelian surface for $t \neq 0)$, the monodromy representation

$$
\pi_{1}(\Delta \backslash\{0\}) \rightarrow \operatorname{Sp}(2, \mathbb{Z})
$$

corresponds to a $\operatorname{Sp}(2, \mathbb{R})$-conjugacy class of elements of finite order in $\operatorname{Sp}(2, \mathbb{Z})$; moreover, any such class corresponds to such a degeneration (see [U1, §1] for details). Wishing to classify all degenerations $\mathscr{A} \rightarrow \Delta$ as above, Ueno uses the classification of such conjugacy classes and their fixed points [U1, §2]. There are fifty-six such classes, of which Ueno treats twenty-six (types I, II, and III), which we refer to as Ueno degenerations. For each conjugacy class, Ueno constructs a degeneration $\mathscr{B} / G \rightarrow \Delta$ where $\mathscr{B}$ is nonsingular and $G$ is a finite cyclic group with fixed points only over $0 \in \Delta$, so $\mathscr{B} / G$ has cyclic quotient singularities. Ueno then resolves the singularities $\mathscr{A} \rightarrow \mathscr{B} / G$ using a procedure 
he calls "canonical resolution". By knowing the action of $G$ on $\mathscr{B}$ and using his procedure for resolving singularities, Ueno is able to explicitly determine the singular fiber $\mathscr{A}_{0}$, which is of the form

$$
m \Theta+\text { components of smaller multiplicities }
$$

where $m=|G|$ and $\Theta$ is the proper transform of $\left((\mathscr{B} / G)_{0}\right)_{\text {red }}$, which is irreducible, since $\mathscr{B}_{0}$ is irreducible.

A minimal model $\mathscr{A}_{\min }$ of $\mathscr{A} \rightarrow \Delta$ is characterized by the following two properties: first, $m K_{\mathscr{A}_{\min }} \sim 0$ for some $m \geq 1$ and second, $\mathscr{A}_{\min }$ has at worst terminal singularities. Since the canonical divisor of the general fiber of $\mathscr{B} / G \rightarrow \Delta$ is trivial, $K_{\mathscr{B} / G}$ is concentrated on $(\mathscr{B} / G)_{0}$, which is irreducible. Since $(\mathscr{B} / G)_{0}$ is a fiber, $(\mathscr{B} / G)_{0} \sim 0$, whereas $(\mathscr{B} / G)_{0} \sim m\left((\mathscr{B} / G)_{0}\right)_{\text {red }}$, so clearly $m K_{\mathscr{B} / G} \sim 0$. Thus we have $\mathscr{A} \rightarrow \mathscr{B} / G$ where, on the one hand, $m K_{\mathscr{B} / G} \sim 0$, so $\mathscr{B} / G$ satisfies the first condition of minimal models, while $\mathscr{A}$ is nonsingular and so in particular has at worst terminal singularities and so satisfies the second condition of minimal models. Clearly if $\mathscr{B} / G$ has only terminal singularities, then $\mathscr{A}_{\text {min }}=\mathscr{B} / G$, while if $m K_{\mathscr{A}_{\min }} \sim 0$, then $\mathscr{A}_{\text {min }}=\mathscr{A}$-in general, one might expect the result to be that $\mathscr{A}_{\min }$ is either $\mathscr{B} / G, \mathscr{A}$ or a partial resolution of $\mathscr{B} / G$. In point of fact, for Ueno's degenerations, we have the following:

1.1. Theorem. For Ueno's degenerations, there is a minimal model $\mathscr{A}_{\min }$ for which $m K_{\mathscr{A}_{\min }} \sim 0$ for some $m \geq 1$ and $\mathscr{A}_{\min }$ has at most terminal singularities. Moreover, either

(i) $\mathscr{A}_{\min }=\mathscr{B} / G$ so $\left(\mathscr{A}_{\min }\right)_{0}$ is irreducible, or

(ii) $\mathscr{A}_{\min }$ is a partial resolution of $\mathscr{B} / G$ dominated by $A$ (so that there are maps $\mathscr{A} \rightarrow \mathscr{A}_{\min } \rightarrow \mathscr{B} / G$, neither of which is an isomorphism) and the map $\mathscr{A} \rightarrow \mathscr{A}_{\min }$ only contracts $\mathbb{P}^{2} s$ to singularities of type $\frac{1}{2}(1,1,1)$, or

(iii) $\mathscr{A}_{\min }=\mathscr{A}$ (that is, the "canonical resolution" of $\mathscr{B} / G$ is the minimal model ), or

(iv) $\mathscr{A}_{\min }$ is obtained from $\mathscr{A}$ by a composition of smooth contractions, contractions of $\mathbb{P}^{2}$ 's to $\frac{1}{2}(1,1,1)$-singularities and simple flips (see 0.3$)$. In this case, $\mathscr{B} / G$ has noncanonical singularities and so in passing to $A_{\min }, \Theta$ must be blown down. The model $\mathscr{A}_{\min }$ is either nonsingular or has only $\frac{1}{2}(1,1,1)$-singularities.

1.2. Note. The singularities of $\mathscr{B} / G$ are canonical if and only if $\mathscr{B} / G$ falls in cases (i), (ii), or (iii) of Theorem 1.1. In particular, in case (iv), $\Theta$ must be contracted. The singularities of $\mathscr{B} / G$ are terminal, terminal and canonical, and canonical only, for cases (i), (ii), and (iii), respectively.

We have illustrated the way in which Ueno's cases break up into our four alternatives in Tables 1, 2, 3, and 4 .

Proof of 1.1. Ueno enumerates his degenerations on p. 64, [U1], for numbers 1-11, and on p. 166, [U2], for numbers 12-23 (note that a few of the twenty-six cases yield similar albeit not isomorphic $\mathscr{A}_{0}$ ), and describes the singular fibers, $\mathscr{A}_{0}$. Of these cases, seven correspond to the most obvious type of degeneration of principally polarized abelian surfaces with finite monodromy, 
TABLE 1 . Cases with $\mathscr{A}_{\min }=\mathscr{B} / G$.

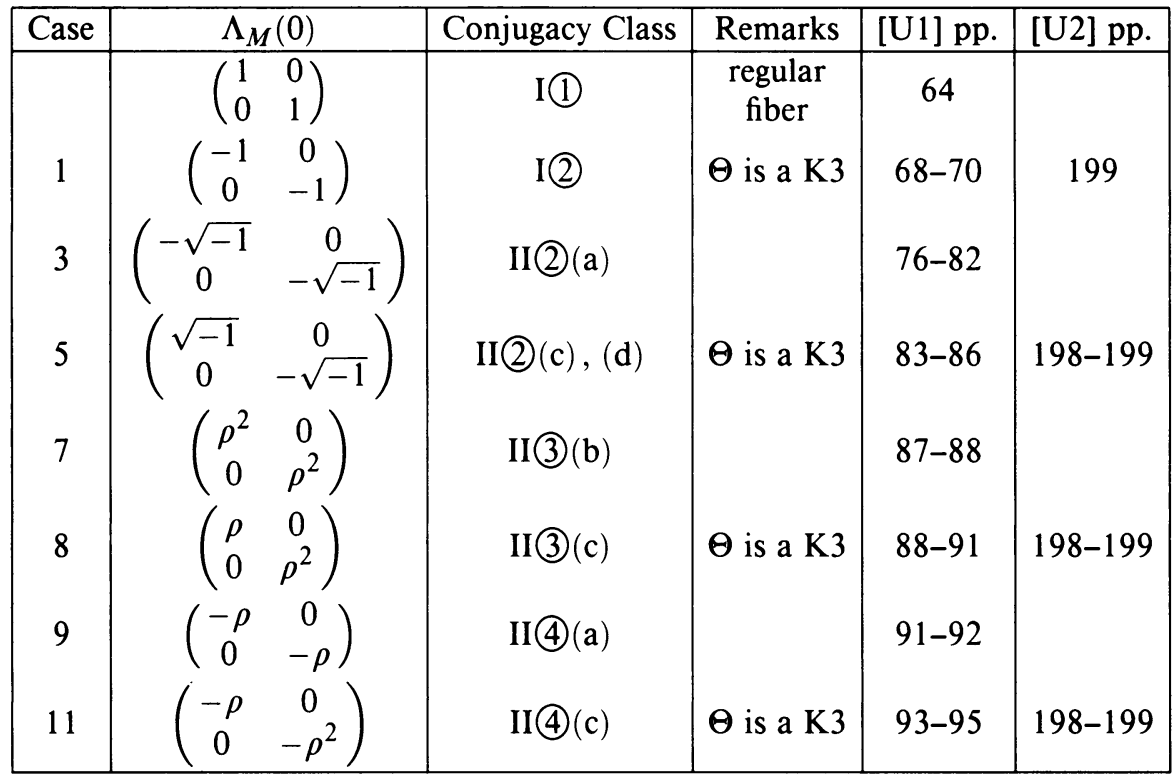

TABLE 2. Cases with $\mathscr{A}_{\text {min }} \rightarrow \mathscr{B} / G$ a partial desingularization.

\begin{tabular}{|c|c|c|c|c|c|}
\hline Case & $\Lambda_{M}(0)$ & Conjugacy Class & Remarks & [U1] pp. & [U2] pp. \\
\hline 12 & $\left(\begin{array}{cc}-\rho & 0 \\
0 & -1\end{array}\right)$ & III(1)(a) & & & $172-177^{*}$ \\
23 & $\left(\begin{array}{cc}-\sqrt{-1} & 0 \\
0 & -1\end{array}\right)$ & III(6)(b) & & & $195-197$ \\
\hline
\end{tabular}

*This case is discussed further in Appendix 1 of this paper.

TABLE 3. Cases with $\mathscr{A}_{\min }=\mathscr{A}$.

\begin{tabular}{|c|c|c|c|c|c|}
\hline Case & $\Lambda_{M}(0)$ & Conjugacy Class & Remarks & [U1] pp. & [U2] pp. \\
\hline 2 & $\left(\begin{array}{cc}1 & 0 \\
0 & -1\end{array}\right)$ & II(1)(a), (b) & $\operatorname{Kod}\left(I_{0}^{*}\right)$ & $70-76$ & 198 \\
6 & $\left(\begin{array}{cc}\rho & 0 \\
0 & \rho\end{array}\right)$ & II(3)(a) & & $86-87$ & \\
15 & $\left(\begin{array}{cc}\rho^{2} & 0 \\
0 & 1\end{array}\right)$ & III(2)(b) & $\operatorname{Kod}\left(\mathrm{IV}^{*}\right)$ & & 169 \\
16 & $\left(\begin{array}{cc}\rho & 0 \\
0 & -1\end{array}\right)$ & III(3)(a) & & & $182-187$ \\
18 & $\left(\begin{array}{cc}-\rho & 0 \\
0 & 1\end{array}\right)$ & III(4)(a) & $\operatorname{Kod}\left(\mathrm{II}^{*}\right)$ & & 169 \\
20 & $\left(\begin{array}{cc}-\sqrt{-1} & 0 \\
0 & 1\end{array}\right)$ & III(5)(a) & $\operatorname{Kod}\left(\mathrm{III}^{*}\right)$ & & 170 \\
22 & $\left(\begin{array}{cc}\sqrt{-1} & 0 \\
0 & -1\end{array}\right)$ & III(6)(a) & & & $191-195$ \\
\hline
\end{tabular}


TABLE 4. Cases in which $\Theta$ eventually blows down.

\begin{tabular}{|c|c|c|c|c|c|}
\hline Case & $\Lambda_{M}(0)$ & Conjugacy Class & Remarks & [U1] pp. & [U2] pp. \\
\hline 4 & $\left(\begin{array}{cc}\sqrt{-1} & 0 \\
0 & \sqrt{-1}\end{array}\right)$ & II (2)(b) & & $82-83$ & \\
10 & $\left(\begin{array}{cc}-\rho^{2} & 0 \\
0 & -\rho^{2}\end{array}\right)$ & II (4)(b) & & $92-93$ & \\
13 & $\left(\begin{array}{cc}-\rho^{2} & 0 \\
0 & -1\end{array}\right)$ & III (1)(b) & & & $178-182^{*}$ \\
14 & $\left(\begin{array}{cc}\rho & 0 \\
0 & 1\end{array}\right)$ & III (2)(a) & $\operatorname{Kod}(\mathrm{IV})$ & & $166-167$ \\
17 & $\left(\begin{array}{cc}\rho^{2} & 0 \\
0 & -1\end{array}\right)$ & III (3)(b) & & & $187-191$ \\
19 & $\left(\begin{array}{cc}-\rho^{2} & 0 \\
0 & 1\end{array}\right)$ & III (4)(b) & $\operatorname{Kod}(\mathrm{II})$ & & $169-170$ \\
21 & $\left(\begin{array}{cc}\sqrt{-1} & 0 \\
0 & 1\end{array}\right)$ & III (5)(b) & $\operatorname{Kod}(\mathrm{III})$ & & 171 \\
\hline
\end{tabular}

*This case is discussed further in Appendix 1 of this paper.

namely the fiber product of a fixed elliptic curve with a degeneration of elliptic curves having finite monodromy. By Kodaira's classification, these are $\mathrm{I}_{0}^{*}, \mathrm{IV}, \mathrm{IV}^{*}, \mathrm{II}^{*}, \mathrm{II}, \mathrm{III}^{*}$ and III, which correspond to numbers $2,14,15$ and $18-21$, respectively, of Ueno's lists. In cases $\mathrm{I}_{0}^{*}, \mathrm{IV}^{*}, \mathrm{II}^{*}$ and $\mathrm{III}^{*}, K_{\mathscr{A}} \sim 0$, so these all satisfy case (iii) of the theorem, while in cases IV, II and III, $\mathscr{A}$ corresponds to the normalized degenerations and so the (smooth) contraction of $\Theta$ gives $\mathscr{A}_{\min }$ as the fiber product of a fixed elliptic curve with the minimal models for IV, II and III (case (iv)).

In an additional seven cases, it may be easily verified that all the singularities of $\mathscr{B} / G$ are terminal, and so these satisfy case (i) of the theorem. This occurs in cases 1, 3, 5, 7-9 and 11. For example, consider case 9, which Ueno treats on pp. 91-92 of [U1]. As Ueno notes on p. $92, \mathscr{B} / G$ has precisely ten singular points, one of type $\frac{1}{6}(1,5,5)$, four of type $\frac{1}{3}(1,2,2)$ and five of type $\frac{1}{2}(1,1,1)$, all of which are easily seen to be terminal by 0.2 .

For the remaining nine cases, it is helpful to compute $m K_{\mathscr{A}}$. Since $m K_{\mathscr{A}_{1}} \sim 0$ for $t \neq 0, m K_{\mathscr{A}}$ is concentrated on $\mathscr{A}_{0}$ and $\mathscr{A}_{0} \sim 0$, so the multiplicities of

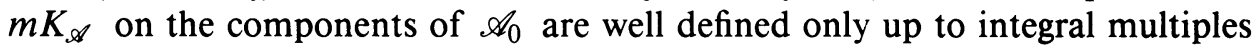
of $\mathscr{A}_{0}$. To fix notation, we may therefore choose a representative of $m K_{\mathscr{A}}$ for which the coefficient of $\Theta$ is zero. For this choice, let $m K_{\mathscr{A}} \sim \sum r_{i} V_{i}$ for $r_{i} \in$ $\mathbb{Z}$. Note that $r_{i}>0$ for all $i$ if and only if $\mathscr{B} / G$ has only terminal singularities; $r_{i} \geq 0$ for all $i$ if and only if $\mathscr{B} / G$ has only canonical singularities and so if $r_{j}<0$ for some $j$, then the singularities of $\mathscr{B} / G$ are not even canonical. The idea behind the rest of the proof is to blow down those $V_{i}$ for which $r_{i}>0$, leave alone those $V_{i}$ for which $r_{i}=0$ and, if $r_{j}<0$ for some $V_{j}$, then blow down $\Theta$, which may require some birational modifications before it can be blown down to a threefold with only terminal singularities.

To compute the coefficients of $m K_{\mathscr{A}}$, we use the genus formula (multiplied by $m)$ applied to curves in $\mathscr{A}_{0}: m(2 g(C)-2)=\left(m K_{\mathscr{A}}\right) \cdot C+m\left(\operatorname{deg} N_{C / \mathscr{A}}\right)$. For example, if $C$ is a double curve, $C \subset V_{1} \cap V_{2}$, where $V_{1}$ and $V_{2}$ meet normally, 
then using Ueno's descriptions of $\mathscr{A}_{0}$, we can determine the genus of $C$ and value of $C \cdot V_{i}$ for all $i$ (note that $C \cdot V_{1}=\left(C^{2}\right)_{V_{2}}$ and $C \cdot V_{2}=\left(C^{2}\right)_{V_{1}}$ ). Now $\operatorname{deg} N_{C / \mathscr{A}}=C \cdot V_{1}+C \cdot V_{2}$, so if $m K_{\mathscr{A}} \sim \sum r_{i} V_{i}$, then the genus formula applied to each such double curve $C$ gives a collection of linear relations between the $r_{i}$ 's.

There are three cases for which $m K_{\mathscr{A}} \sim 0$ (cases 6,16 and 22) - these satisfy case (iii) of the Theorem. Ueno's case 6 is illustrated on p. 66 and treated on pp. 86-87 of [U1]. Here $\mathscr{A}_{0}=3 \Theta+\sum_{\nu=1}^{9} \Theta_{\nu}$ where the $\Theta_{\nu}$ correspond to resolutions of singularities of type $\frac{1}{3}(1,1,1)$. Let $3 K_{\mathscr{A}} \sim \sum r_{\nu} \Theta_{\nu}$, since $r(\Theta)=0$ by assumption. Now if $\theta_{\nu}=\Theta \cap \Theta_{\nu}$, then $\theta_{\nu}$ is a line on $\Theta_{\nu} \cong \mathbb{P}^{2}$ and $\left(\theta_{\nu}^{2}\right)_{\boldsymbol{\Theta}}=-3$, so $\theta_{\nu} \cdot \Theta=1, \theta_{\nu} \cdot \Theta_{\nu}=-3, g\left(\theta_{\nu}\right)=0$ and $\operatorname{det} N_{\theta_{\nu} / \mathscr{A}}=-2$ so by the genus formula, $0=\left(3 K_{\mathscr{A}}\right) \cdot \theta_{\nu}=r_{\nu} \theta_{\nu} \cdot \Theta_{\nu}=-3 r_{\nu}$ so $r_{\nu}=0$. Thus $3 K_{\mathscr{A}} \sim 0$. In case $16, \mathscr{A}_{0}$ is a somewhat complicated collection of surfaces (illustrated on p. 169 of [U2]). Letting $6 K_{\mathscr{A}} \sim \sum r_{i} \Theta_{i}+\sum r_{i j} \Theta_{i j}$, it is not difficult to show that each $r_{i}$ and $r_{i j}$ is zero using double curves as in the previous paragraph. Note that in this case all double curves $C$ are rational and if $C=V \cap W$, then $\left(C^{2}\right)_{V}=0$ and $\left(C^{2}\right)_{W}=-2$ (for some choice of $V$ and $W$ ), making the calculation quite simple. In case 22 (illustrated on p. 172 of [U2]), all double curves are as in case 16, except for $C=\Theta \cap \Theta_{3}$ : thus it is easy to show that $4 K_{\mathscr{A}} \sim r_{3} \Theta_{3}$. Now $g(C)=1$ and $\left(C^{2}\right)_{\boldsymbol{\Theta}}=\left(C^{2}\right)_{\boldsymbol{\Theta}_{3}}=0$ so $\left(4 K_{\mathscr{A}}\right) \cdot C$ gives no information about $r_{3}$. Let $F$ be a fiber on $\Theta_{3}$, which is a minimal ruled surface over $C$. Now by the exactness of

$$
\left.0 \rightarrow N_{F / \boldsymbol{\Theta}_{3}} \rightarrow N_{F / \mathscr{A}} \rightarrow N_{\boldsymbol{\Theta}_{3} / \mathscr{A}}\right|_{F} \rightarrow 0,
$$

since $\left(F^{2}\right)_{\Theta_{3}}=0$ and $F \cdot \Theta_{3}=F \cdot(-2 \Theta)=-2$ (because $\mathscr{A}_{0} \sim 0$ ), $\operatorname{det} N_{F / \mathscr{A}}=$ -2 , so $\left(4 K_{\mathscr{A}}\right) \cdot F=0$ and so $r_{3}=0$.

There are two cases (numbers 12 and 23) for which $\mathscr{A}_{\min }$ is obtained from $\mathscr{A}$ by contracting $\mathbb{P}^{2}$ 's to singularities of type $\frac{1}{2}(1,1,1)$-these satisfy case (ii) of the Theorem. In each of these cases, using the same techniques for computing $m K_{\mathscr{A}}$ as above, $m K_{\mathscr{A}} \sim \sum r \Theta_{i j}$, where $r=3$ for number 12 and $r=2$ for number 23 (using Ueno's notations-see pp. 167 and 173 of [U2]), where $\boldsymbol{\Theta}_{i j} \cong \mathbb{P}^{2} ;$ moreover for each $(i, j), N_{\Theta_{i j} / \mathscr{A}} \cong \mathscr{O}_{\mathbb{P}^{2}}(-2)$, so all the $\boldsymbol{\Theta}_{i j}$ 's are contractible to terminal points of type $\frac{1}{2}(1,1,1)$. Note that in case 12 , there are in fact four more such $\mathbb{P}^{2}$ 's than indicated by Ueno (please see Appendix 1), which does not affect our results here.

The final four cases (numbers 4, 10,13 and 17) are the most interesting and satisfy case (iv) of the Theorem. In case 17, as Ueno notes (p. 190 of [U2]), $\Theta$ is smoothly contractible to a curve, after which $\mathscr{A}_{\min }$ is obtained by contracting all the $\boldsymbol{\Theta}_{i j}$ 's to terminal points of type $\frac{1}{2}(1,1,1)$ as in the above paragraph. In each of the three remaining cases some component of $\mathscr{A}_{0}$ has negative multiplicity in the choice of representative of $m K_{\mathscr{A}}$ for which $\Theta$ has multiplicity zero, requiring that $\Theta$ be contracted; however $\Theta$ is not smoothly contractible and in fact $\rho(\Theta)>2$. Passing from $\mathscr{A}$ to $\mathscr{A}^{\prime}$ by contracting all $\mathbb{P}^{2}$ 's having -2 as the degree of the normal bundle to $\frac{1}{2}(1,1,1)$-singularities and using 0.5 we find an exceptional curve $\widetilde{C} \subset \Theta$. Using information from $N_{\Theta / \mathscr{A}}$, we show that $N_{\widetilde{C} / \mathscr{A}} \cong \mathscr{O}(-1) \oplus \mathscr{O}(-1)$ and that $\widetilde{C}$ meets (in a single point) a component $\Theta_{\nu}$ of $\mathscr{A}_{0}$ where $\Theta_{\nu} \cong \mathbb{P}^{2}$ and $N_{\Theta_{\nu} / \mathscr{A}} \cong \mathscr{O}_{\mathbb{P}^{2}}(-2)$, allowing 
the contraction and simple flip of 0.3 to be applied to $\widetilde{C}$ (or, equivalently, the simple flip of 0.3 may be applied to the image of $\widetilde{C}$ in $\mathscr{A}^{\prime}$-note that such a flip decreases the number of $\frac{1}{2}(1,1,1)$-singularities by one). We continue finding such $\widetilde{C}$ until there are no more singular points in $\mathscr{A}^{\prime}$, at which point $\Theta$ proves to be minimal ruled and is then smoothly contractible, yielding $\mathscr{A}_{\text {min }}$. We treat each of the three cases separately.

In case $4, \mathscr{A}_{0}=4 \Theta+\sum_{\nu=1}^{4} \Theta_{\nu}+\sum_{\nu=5}^{10} 2 \Theta_{\nu}$ where $\Theta_{\nu} \cong \mathbb{P}^{2}$ for all $\nu$. Now

$$
4 K_{\mathscr{A}} \sim \sum_{\nu=1}^{4}-\Theta_{\nu}+\sum_{\nu=5}^{10} 2 \Theta_{\nu} .
$$

Let $\theta_{\nu}=\Theta \cap \Theta_{\nu}$-each $\theta_{\nu}$ is a smooth rational curve. For $\nu \leq 4,\left(\theta_{\nu}^{2}\right)_{\Theta}=-4$, while for $\nu \geq 5,\left(\theta_{\nu}^{2}\right)_{\boldsymbol{\theta}}=-2$. Since $\mathscr{A}_{0} \sim 0$, we may compute $N_{\boldsymbol{\Theta} / \mathscr{A}} \sim \boldsymbol{\Theta} \cdot \boldsymbol{\Theta}$ and so, by adjunction, we may also compute $K_{\Theta}$ :

$$
4 N_{\boldsymbol{\Theta} / \mathscr{A}} \sim-\left(\theta_{1}+\cdots+\theta_{4}\right)-2\left(\theta_{5}+\cdots+\theta_{10}\right)
$$

and

$$
4 K_{\boldsymbol{\Theta}} \sim-2\left(\theta_{1}+\cdots+\theta_{4}\right) .
$$

Let $\mathscr{A} \rightarrow \mathscr{A}^{\prime}$ contract the $\Theta_{\nu}$ for $\nu=5, \ldots, 10$ to singular points of type $\frac{1}{2}(1,1,1)$. Let $\psi$ be the restriction of $\mathscr{A} \rightarrow \mathscr{A}^{\prime}$ to $\Theta$, so $\psi: \Theta \rightarrow S$ (where $S=\psi(\Theta)$ ) contracts the six $(-2)$-curves $\theta_{5}, \ldots, \theta_{10}$. Now $4 K_{S} \sim-2 D$ where $D=\psi\left(\theta_{1}\right)+\cdots+\psi\left(\theta_{4}\right)$, so $K_{S}$ is not numerically effective. Now $\Theta$ is rational and $K_{\Theta}^{2}=-4$, so $\rho(\Theta)=14$ and so $\rho(S)=8>2$. By 0.5 , there is a $C \subset S$ with $C^{2}<0$ such that $\tilde{C}$ is an exceptional curve on $\Theta$, for $\tilde{C}$ the proper transform of $C$ under $\psi^{-1}$. Now $\psi$ contracts only (-2)-curves, so by 0.6 ,

$$
\left(C^{2}\right)_{S} D=\left(\widetilde{C}^{2}\right)_{\boldsymbol{\Theta}}+\frac{1}{2} \sum_{\nu=5}^{10}\left(\widetilde{C} \cdot \theta_{\nu}\right) .
$$

Since $\left(\widetilde{C}^{2}\right)_{\boldsymbol{\Theta}}=-1, \sum_{\nu=5}^{10}\left(\tilde{C} \cdot \theta_{\nu}\right) \leq 1$. Now $\tilde{C} \cdot N_{\Theta / \mathscr{A}} \in \mathbb{Z}$, so in fact $\sum_{\nu=5}^{10} \widetilde{C} \cdot \theta_{\nu}=1$. Thus $\widetilde{C}$ meets exactly one $\Theta_{\nu}$ for some $\nu, 5 \leq \nu \leq 10$; moreover $\left(\widetilde{C}^{2}\right)_{\Theta}=-1$ and $\widetilde{C} \cdot N_{\Theta / \mathscr{A}}=\tilde{C} \cdot \Theta=-1$, so, since $\widetilde{\widetilde{C}}$ is rational, $N_{\widetilde{C} / \mathscr{A}} \cong \mathscr{O}_{\widetilde{C}}(-1) \oplus \mathscr{O}_{\widetilde{C}}(-1)$; thus $\widetilde{C}$ satisfies the conditions of 0.3 . The contraction and simple flip of 0.3 drops $\rho(\Theta)$ by 2 (since $\widetilde{C}$ and one $\theta_{\nu}$, $5 \leq \nu \leq 10$, are contracted) and contracts one $\Theta_{\nu}, 5 \leq \nu \leq 10$; otherwise $\mathscr{A}_{0}$ is unchanged. Similarly the simple flip drops $\rho(S)$ by 1 and decreases the number of $\frac{1}{2}(1,1,1)$-singular points from 6 to 5 . Repeating these operations five more times, all $\Theta_{\nu}, \nu=5, \ldots, 10$, are contracted and the resulting $\Theta$ is a smooth minimal ruled surface in a nonsingular threefold. For $F$ a fiber on $\Theta, F \cdot K_{\Theta}=-2$, so $F \cdot\left(\theta_{1}+\cdots+\theta_{4}\right)=4$ and therefore $\left.N_{\Theta / \mathscr{A}}\right|_{F}=F \cdot \Theta=-1$, so $\Theta$ is smoothly contractible, leaving a degeneration $\mathscr{C} \rightarrow \Delta$ where $\mathscr{C}$ is nonsingular, $\mathscr{C}_{0}=\bar{\Theta}_{1}+\cdots+\bar{\Theta}_{4}$, the images of the corresponding $\Theta_{\nu}$ 's; moreover,

$$
4 K_{\mathscr{C}} \sim-\left(\bar{\Theta}_{1}+\cdots+\bar{\Theta}_{4}\right) \sim-\mathscr{C}_{0} \sim 0
$$

so $\mathscr{C}=\mathscr{A}_{\min }$ as claimed in (iv) of the theorem.

In case $10, \mathscr{A}_{0}=6 \Theta+\Theta_{1}+\sum_{\nu=2}^{5} 2 \Theta_{\nu}+\sum_{\nu=6}^{10} 3 \Theta_{\nu}$ where $\Theta$ is a rational surface and $\Theta_{\nu} \cong \mathbb{P}^{2}$ for all $\nu$. Arguing as in case 4 , we have

$$
6 N_{\mathbf{\Theta} / \mathscr{A}} \sim-\widetilde{D}_{a}-2 \widetilde{D}_{b}-3 E
$$




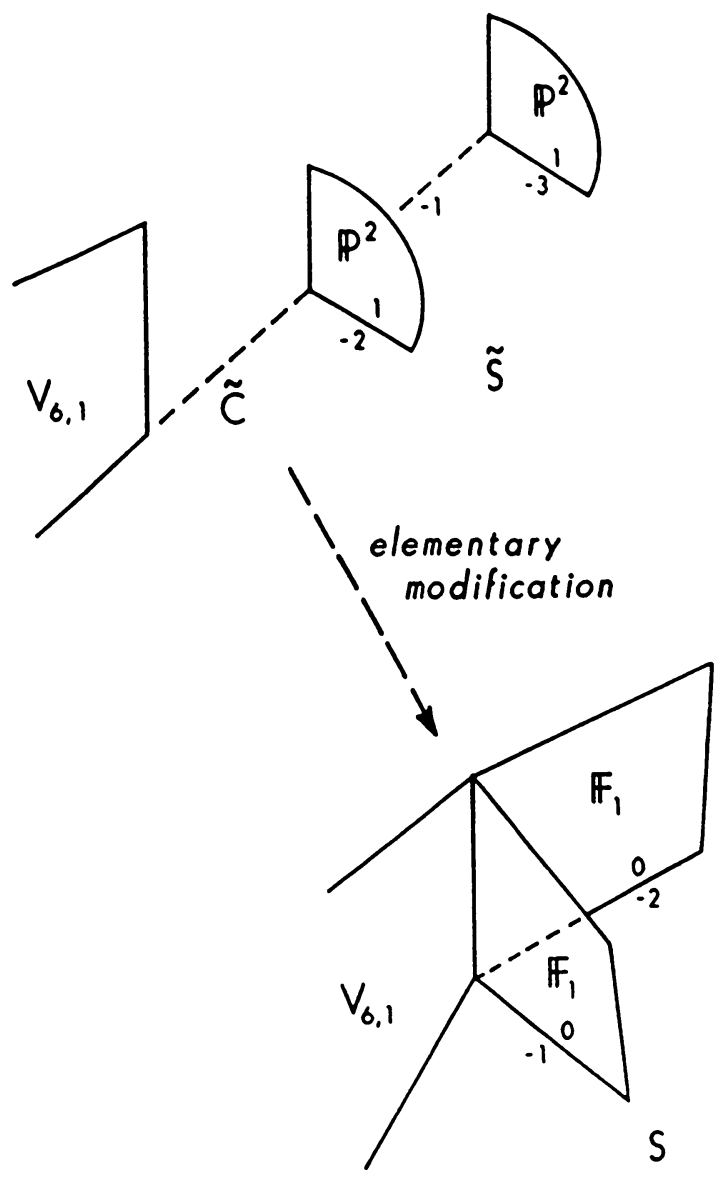

FIGURE 1. One view of $\tilde{\varphi}$

where $\widetilde{D}_{a}=\Theta \cap \Theta_{1}, \widetilde{D}_{b}=\sum_{\nu=2}^{5} \Theta \cap \Theta_{\nu}$ and $E=\sum_{\nu=6}^{10} \Theta \cap \Theta_{\nu}$, and

$$
6 K_{\Theta} \sim-4 \widetilde{D}_{a}-2 \widetilde{D}_{b}
$$

Again, let $\psi: \Theta \rightarrow S$ contract the five $(-2)$-curves in $E$ (induced from the contraction of the $\Theta_{\nu}$ 's, $\left.\nu=6, \ldots, 10\right)$, so $6 K_{S} \sim-4 D_{a}-2 D_{b}$ where $D_{i}=$ $\psi\left(\widetilde{D}_{i}\right)$. Since $K_{\Theta}^{2}=-4, \rho(\Theta)=14$ and $\rho(S)=9>2$. As in case 4, there is an exceptional curve $\widetilde{C} \subset \Theta$ such that $\widetilde{C} \cdot N_{\Theta / \mathscr{A}}=\widetilde{C} \cdot \Theta=1$ and $\widetilde{C} \cdot \widetilde{D}_{a}=\widetilde{C} \cdot \widetilde{D}_{b}=\widetilde{C} \cdot E=1$. Since, for $\nu \geq 6, N_{\Theta_{\nu} / \mathscr{A}} \cong \mathscr{O}_{\mathbb{P}^{2}}(-2), \widetilde{C}$ satisfies the conditions for contraction and simple flip of 0.3 . Renumbering, assume that $\widetilde{C} \cdot \Theta_{10}=\tilde{C} \cdot \Theta_{5}=1$. After a contraction and simple flip, $\Theta_{5}$ will be isomorphic to an $\mathbb{F}_{1}$ meeting $\Theta$ along a fiber on $\Theta_{5}$ which is an exceptional curve on $\Theta$, so in fact $\Theta_{5}$ will be smoothly contractible (to a curve contained in $\left.\Theta_{1}\right)$.

Let $\tilde{\varphi}$ be the composition of the contraction, simple flip and contraction described above. Figure 1 shows the beginning of $\tilde{\varphi}$ as an elementary modification centered at $\widetilde{C}$, after which one easily sees that the two $\mathbb{F}_{1}$ 's are smoothly contractible to a curve. After $\tilde{\varphi}$ is applied to $\mathscr{A}$, the new degeneration is 
still nonsingular, $\Theta_{5}$ and $\Theta_{10}$ have been contracted, $\rho(\Theta)$ has been reduced by 3 and $\rho(S)$ by 2 . Iterating the $\tilde{\varphi}$ procedure four more times produces a new nonsingular degeneration $\mathscr{C} \rightarrow \Delta$ for which $\mathscr{C}_{0}=6 \bar{\Theta}+\bar{\Theta}_{1}+3 \bar{\Theta}_{6}$ where $\bar{\Theta}_{6} \cong \mathbb{P}^{2}, \rho(\bar{\Theta})=2$, so $\bar{\Theta}$ is minimal rational ruled (in fact, $\bar{\Theta} \cong \mathbb{F}_{2}$ since $\left.\left(\left(\bar{\Theta} \cap \bar{\Theta}_{6}\right)^{2}\right)_{\boldsymbol{\Theta}}=-2\right)$ and $6 K_{\bar{\Theta}} \sim-4 \bar{D}_{a}$. As in case 4 , it is easy to show that $\bar{\Theta}$ is smoothly contractible to $\bar{\Theta} \cap \bar{\Theta}_{6}$, after which $\bar{\Theta}_{6}$ is smoothly contractible to a point, leaving a new nonsingular degeneration $\mathscr{D} \rightarrow \Delta$ where $\mathscr{D}_{0}$ is the image of $\bar{\Theta}_{1}$. Thus $\mathscr{D}_{0}$ is irreducible and so $6 K_{\mathscr{D}} \sim 0$. Note that the singular point of $\mathscr{D}_{0}$ is bad: $\bar{\Theta} \cap \bar{\Theta}_{1}$ is a trisection on $\bar{\Theta} \cong \mathbb{F}_{2}$ of genus four and the two smooth contractions $\mathscr{C} \rightarrow \mathscr{D}$ restricted to $\bar{\Theta}_{1}$ are the minimal desingularization of $\mathscr{D}_{0}$.

In our final case, number 13 , we proceed in a manner completely analogous to that used in case 4 . For this case, $\mathscr{A}_{0}=6 \Theta+2 \Theta_{1}+\sum_{i=1}^{4} \Theta_{2 i}+2 \Theta_{3}+3 \sum_{i=4}^{7} \Theta_{i}$ where $\Theta$ is rational and, for $4 \leq i \leq 7, \Theta_{i} \cong \mathbb{P}^{2}$ with $N_{\Theta_{i} / \mathscr{A}} \cong \mathscr{O}_{\mathbb{P}^{2}}(-2)$. Note that, as in case 12, the four $\mathbb{P}^{2}$ 's $\Theta_{4}, \ldots, \Theta_{7}$ are not in Ueno's paper (please see Appendix 1). Computing as before,

$$
6 K_{\mathscr{A}} \sim-2 \Theta_{1}-\sum_{i=1}^{4} \Theta_{2 i}-2 \Theta_{3}+3 \sum_{i=4}^{7} \Theta_{i} .
$$

Arguing as in case 4 , there is an exceptional curve $\tilde{C} \subset \Theta$ where $\tilde{C} \cdot \sum_{i=4}^{7} \Theta_{i}=1$ and $\tilde{C} \cdot \Theta=-1$, so $\tilde{C}$ satisfies the conditions of 0.3 . A contraction and simple flip centered at $\widetilde{C}$, followed by three more, contracts $\Theta_{4}, \ldots, \Theta_{7}$ and leaves $\Theta$ minimal ruled and smoothly contractible to a curve. That contraction produces a nonsingular degeneration $\mathscr{C} \rightarrow \Delta$ for which $\mathscr{C}_{0}=2 \bar{\Theta}_{1}+\sum_{i=1}^{4} \bar{\Theta}_{2 i}+2 \bar{\Theta}_{3}$ and

$$
6 K_{\mathscr{C}} \sim-2 \overline{\boldsymbol{\Theta}}_{1}-\sum_{i=1}^{4} \overline{\boldsymbol{\Theta}}_{2 i}-2 \overline{\boldsymbol{\Theta}}_{3} \sim-\mathscr{C}_{0} \sim 0,
$$

so $\mathscr{C}=\mathscr{A}_{\min }$.

\section{TRIPLE-POINT-FREE DEGENERATIONS OF $\kappa=0$ SURFACES}

In this section, we briefly recall the definition and properties of triple-pointfree degeneration of $\kappa=0$ surfaces from [CM1], indicate our desired result and outline its proof.

2.1. Definitions. By a degeneration of surfaces, we mean $\pi: Y \rightarrow \Delta$ where $Y$ is a normal three-dimensional complex space, $\Delta$ is a unit disk in $\mathbb{C}$ and $\pi$ is a proper surjective holomorphic map. In addition, we assume that $Y_{t}=\pi^{-1}(t)$ is nonsingular for $t \neq 0$ and that all components of $Y_{0}$ are Moishezon surfaces. By a triple-point-free degeneration of $\kappa=0$ surfaces, we mean a degeneration of surfaces for which $Y$ is nonsingular, for $t \neq 0, Y_{t}$ is a surface with $\kappa=0$ and $Y_{0}$ is a union of nonsingular surfaces meeting normally; moreover, no three distinct components of $Y_{0}$ intersect. Such a degeneration is birationally minimal if there is no component of $Y_{0}$ which may be smoothly contracted preserving the above assumptions. Note that birationally minimal does not necessarily imply minimal in the sense of minimal models. 
For $\pi: Y \rightarrow \Delta$ a triple-point-free degeneration of $\kappa=0$ surfaces, let $\Gamma$ be the dual graph of $Y_{0}$ (since $Y_{0}$ is a triple-point-free degeneration, $\operatorname{dim} \Gamma=1$ ). The vertices of $\Gamma$ (which correspond to components of $Y_{0}$ ) are assigned weights according to multiplicities in $Y_{0}$ and $K_{Y}$-see p. 354 of [CM1]. We let $\Gamma_{\min }$ be the span of those vertices having absolutely minimal such weights. The components of $Y_{0}$ corresponding to connected components of $\Gamma \backslash \Gamma_{\min }$ are flowers. If $\Gamma_{\min }$ is a single vertex, then the component corresponding to $\Gamma_{\min }$ is a flower pot and $\pi: Y \rightarrow \Delta$ is a flower-pot degeneration. If $\Gamma_{\min }$ has two or more vertices and each vertex of $\Gamma_{\min }$ has at most two edges in $\Gamma_{\min }$, then the components of $Y_{0}$ form a chain or a cycle (and so $\pi: Y \rightarrow \Delta$ is a chain degeneration or a cycle degeneration), according to whether $\Gamma_{\min }$ forms an interval or a circle, respectively.

2.2. Results from [CM1]. In [CM1], we consider $\pi: Y \rightarrow \Delta$, a birationally minimal triple-point-free degeneration of $\kappa=0$ surfaces. Results include

1. $\pi: Y \rightarrow \Delta$ is either a flower pot, chain or a cycle degeneration. This is the Main Theorem [CM1, p. 353].

2. All flowers are classified into 21 types. Each type is completely described geometrically. This is Theorem 3.9 of [CM1]. Please note the corrections to Table 3.4 of [CM1] in our Appendix 2.

3. If $\pi: Y \rightarrow \Delta$ is a flower-pot degeneration for which the pot is irregular or $\pi: Y \rightarrow \Delta$ is a cycle degeneration, then there is $\psi: Y_{0} \rightarrow E$, where $E$ is an elliptic curve, such that every fiber of $\psi$ is a normalized Kodaira elliptic curve [CM1, Theorems 4.2 and 5.3]. In these cases, therefore, there is a smooth minimal model $Z$ obtained from $Y$ by smooth blow-downs, i.e. passing to the (unnormalized) Kodaira elliptic curves-clearly, $K_{Z} \equiv 0$.

4. If $\pi: Y \rightarrow \Delta$ is a chain degeneration, then, after contractions and simple flips $(0.3)$, the components of the chain are elliptic ruled, except possibly those on the ends, which may be rational or elliptic ruled. In addition, there are very strong restrictions on the types of flows in $Y$ [CM1, Theorem 6.7].

5. If $\pi: Y \rightarrow \Delta$ is a flower-pot degeneration for which the pot is $\mathbb{P}^{2}$ or minimal rational ruled, then $Y_{0}$ is completely classified [CM1, Lemmas 3.6, 3.7, and 3.8].

2.3. Note. Knowing the flowers and chain components of chain degenerations, it can be shown that the semistable models for chain degenerations are either chain or cycle degenerations. Similarly, the semistable models for cycle degenerations remain cycle degenerations, so by [Ku and $\mathrm{PP}$ ], the monodromy associated to such degenerations has infinite order-in particular such degenerations do not have "potentially good reduction".

For the remainder of this paper, we will concentrate on flower-pot degenerations. But we would like to point out that chain degenerations may be treated in a similar manner (although these are much easier than flower-pot degenerations) and cycle degenerations are already minimal, since $K_{Y}$ is $\mathbb{Q}$-linearly equivalent to zero.

2.4. Models of flower-pot degenerations: $Y, Z$, and $X$. From 2.1, we know that if $\pi: Y \rightarrow \Delta$ is a flower-pot degeneration, $Y_{0}$ has a distinguished component, the pot, and flowers. As we will see in $\S 3$, the flowers may all be contracted to singular points. Let $\tau: Y \rightarrow Z$ be that contraction, so $Z_{0}$ is irreducible, consisting of the image of the pot, and so $K_{Z} \equiv 0$. Unfortunately, $Z$ need not 
be a minimal model of $Y$, since the singularities on $Z$ are not necessarily terminal. In $\S 3$, we tabulate the contribution each type of flower makes to $K_{Y}$, normalizing the coefficients of $K_{Y}$ so that the coefficient of the pot is zero: thus the contraction of a flower leads to a terminal (resp. canonical) singularity if and only if all coefficients of the flower are positive (resp. nonnegative). Let $\sigma: Y \rightarrow X$ be the contraction of all terminal flowers and subflowers (i.e., all components of flowers with positive coefficients), so clearly $\tau$ factors through $\sigma$ and $X$ represents a partial resolution of $Z$. So we have $Y \stackrel{\sigma}{\rightarrow} X \rightarrow Z$ (with $\tau$ the composite morphism), where $Y$ is nonsingular, $X$ has at most terminal singularities, and $K_{Z} \equiv 0$. Note that in Ueno's degenerations, the rôle of $Y$ is played by $\mathscr{A}$, and that of $Z$ is played by $\mathscr{B} / G$.

2.5. $Q(n), A(n)$ and $P(n)$ singularities. The terminal model $X$ of $Y$ above has terminal singularities of three elementary types: quotient singularities of type $\frac{1}{n+1}(1,1, n)$, which we denote by $Q(n)$, singularities locally described by $x^{2}+y^{2}+z^{2}+t^{2 n+1}=0$ at $(0,0,0,0)$, which we denote by $A(n)$, and those locally described as the quotient of the hypersurface $x^{2}+y^{2}+z^{2}+t^{n}=0$ at $(0,0,0,0)$ by $\mathbb{Z} / 2 \mathbb{Z}$ acting via $\operatorname{diag}(-1,-1,-1,1)$, which we denote by $P(n)$. These terminal singularities will prove to be the only singularities of $Y_{\min }$, the minimal model of $Y$, even when $Y_{\min } \neq X$ ! Note that $Q(1)$ was the only singularity occurring in $\mathscr{A}_{\min }$ for Ueno's degenerations.

2.6. Flips. In $\S 4$, we geometrically construct a collection of elementary flips. These generalize the simple flip of 0.3 .

2.7. Main Theorem. For flower-pot degenerations, there is a minimal model $Y_{\min }$ for which $K_{Y_{\min }} \equiv 0$ and $Y_{\min }$ has at most terminal singularities. Moreover, in the notation above, either

(i) $Y_{\min }=Z$ so $\left(Y_{\min }\right)_{0}$ is irreducible, or

(ii) $Y_{\min }$ is a partial resolution of $Z$ dominated by $Y$ (so that there are maps $Y \rightarrow Y_{\min } \rightarrow Z$, neither of which is an isomorphism) and $Y_{\min }$ has singularities of type $Q(n), A(n)$ and $P(n)$ only, or

(iii) $Y_{\min }=Y$ so $Y_{\min }$ is nonsingular, or

(iv) $Y_{\min }$ is obtained from $Y$ by a composition of divisorial contractions to $Q(n), A(n)$ and/or $P(n)$ singularities, followed by elementary flips (see $\S 4)$. In this case $Y_{\min } \neq X$, but $Y_{\min }$ is yet either nonsingular or has only $Q(n), A(n)$, and $P(n)$ singularities.

2.8. Outline of Proof. If the flowers of $Y_{0}$ lead only to canonical singularities on $Z$, then $Y_{\min }=X$ and the components of $X_{0}$ are the proper transforms of the pot and those flower components with zero contribution to $K_{Y}$. By the classification of flowers and the computation of the canonical bundle contributions for each flower (see Table 6), we see that $Y_{\min }=X$ unless $Y$ has flowers of types $4 B, 6 B, 6 D, 6 \beta, 8 \beta$ or $12 \beta$ (see Proposition 3.7). Thus, in the absence of such flowers, $Y_{\min }=X$ and the theorem is proved with $Y$ satisfying (i), (ii), or (iii), according to whether $X=Z, X \neq Y$, and $X \neq Z$, or $X=Y$, respectively.

$Y$ will satisfy (iv) of the theorem $\left(Y_{\min } \neq X\right)$ if $Y$ has flowers of types $4 B, 6 B, 6 \beta, 8 \beta$ or $12 \beta$. From Table 6 , the table of contributions of flower components to $K_{Y}$ (and so to $K_{X}$ ), since these "bad" flowers have negative 
contribution, $\Theta$, the proper transform of the pot, and all flower components in $X$ with zero contribution as above to $K_{X}$ (i.e. those whose canonical contributions equal that of $\Theta$ ) must all be contracted. In order to accomplish this, flips (constructed in $\S 4$ ) are used to reduce the rank of Pic $\Theta$ until $\Theta$ becomes contractible, after which the rest of the flower components with zero contribution to $K_{X}$ are contracted. This is how $Y_{\min }$ is obtained from $X$. In $\S 4$, the flips are constructed, while in $\S 5$ the rather delicate bookkeeping of the double curves on $\theta$ is explained and the proof completed.

2.9. Note. If we assume that there is a pluricanonical divisor supported on $Y_{0}$ (for example, if $\pi$ is projective, as in Ueno's degenerations), then we can strengthen the conclusion of 2.7 to $m K_{Y_{\min }} \sim 0$ for some $m \geq 1$.

\section{SOME TERMINAL SINGULARITIES AND CONTRACTIONS OF SOME FLOWERS}

In this section, we recall a few properties of certain terminal quotient singularities, and describe how they are related to the "flowers" in a flower-pot degeneration. Throughout this section, $X$ always refers to a threefold.

3.1. $Q(n)$-singularities. We say $X$ has a $Q(n)$-singularity at $Q$ if $(X, Q)$ is a quotient singularity of type $\frac{1}{n+1}(1,1, n)$. Here $Q$ is a singular point if $n \geq 1$; moreover, it is convenient to allow $Q(0)$ as a smooth point. $Q(n)$ singularities, sometimes called Shepherd-Barron nodes, are well understood (for example, see [R3, $\S 5$ with $a=1]) . Q(n)$ is terminal of index $n+1$, and it has a particularly simple resolution which can be described inductively in the following way. For $n \geq 1$ we define the distinguished partial resolution $\tilde{\pi}: \tilde{X} \rightarrow X$ to be a map for which $\tilde{\pi}^{-1}(Q)=\overline{\mathbb{F}}_{n}$, and $\widetilde{X}$ has a singular point of type $Q(n-1)$ at the vertex $\widetilde{Q}$ of $\overline{F_{n}}$ (and is smooth elsewhere). This distinguished partial resolution can be constructed using toroidal embeddings; it is described explicitly in [U1, §4], and [F].

Let $S$ be a Weil divisor on $X$. When $n \geq 1$, we say that $S$ meets $Q$ normally if, for $\tilde{S}$ the proper transform of $S$ under $\tilde{\pi}, \tilde{S} \cdot F=1$ where $F$ is a generator of the cone $\overline{\mathbb{F}}_{n}$. When $n=0$, we say that $S$ meets $Q$ normally if, after blowing up the point $Q$, the proper transform of $S$ meets the exceptional divisor (which is a $\mathbb{P}^{2}$ ) transversely in a line. (In other words, $S$ is smooth at $Q$.) This definition has been designed so that in a distinguished partial resolution, the exceptional divisor meets the new singular point normally. Similarly, for $C$ a curve on $X$, we say that $C$ meets $Q$ normally if $Q$ is a smooth point on $C$ and (when $n \geq 1$ ) the proper transform of $C$ does not pass through $\widetilde{Q}$.

3.2. $A(n)$-singularities. We say $X$ has an $A(n)$-singularity at $P$ if $(X, P)$ is locally analytically isomorphic to the singularity at $(0,0,0,0)$ of the hypersurface in $\mathbb{C}^{4}$ given by $x^{2}+y^{2}+z^{2}+t^{2 n+1}$. Once again we include the smooth point $A(0)$.

The singularities $A(n)$ are compound DuVal singularities (see [R3, §3]), and so are terminal and Gorenstein (index 1). They have particularly simple resolutions which can be described inductively in the following way. For $n \geq 1$ we define the distinguished partial resolution $\tilde{\pi}: \tilde{X} \rightarrow X$ to be the blowup of $X$ at $P$. The exceptional divisor is $\overline{\mathbb{F}}_{2}$, and the vertex $\widetilde{P}$ of $\overline{\mathbb{F}}_{2}$ is the unique singular point on $\widetilde{X}$; it has type $A(n-1)$. 
TABLE 5. Terminal flowers

\begin{tabular}{|c|l|}
\hline flower & singularity \\
\hline $2 A$ & $Q(1)$ \\
$3 A$ & $Q(2)$ \\
$4 A$ & $Q(3)$ \\
$6 A$ & $Q(5)$ \\
$2 B$ & $A(0)^{*}$ \\
$2 C$ & $A(n)^{*}, n \geq 1$ \\
$4 D$ & $P(n)$ \\
\hline
\end{tabular}

Let $S$ be a Weil divisor on $X$. When $n \geq 1$, we say that $S$ meets $P$ normally if, for $\widetilde{S}$ the proper transform of $S$ under $\tilde{\pi}, \widetilde{S} \cdot F=1$ where $F$ is a generator of the cone $\overline{\mathbb{F}}_{2}$. When $n=0$, we say that $S$ meets $P$ normally if, after blowing up the point $P$, the proper transform of $S$ meets the exceptional divisor (which is a $\mathbb{P}^{2}$ ) transversely in a smooth conic. This definition has been designed so that in a distinguished partial resolution, the exceptional divisor always meets the new singular point normally. (This is why we have made a distinction between $Q(0)$ and $A(0)$ : although both are smooth points, the meaning of "meets normally" is different in these two cases.) Similarly, for $C$ a curve on $X$, we say that $C$ meets $P$ normally if $Q$ is a smooth point on $C$ and (when $n \geq 1$ ) the proper transform of $C$ does not pass through $\widetilde{P}$.

3.3. $P(n)$-singularities. We say $X$ has a $P(n)$-singularity at $P$ if $(X, P)$ is locally analytically isomorphic to the quotient of the hypersurface $x^{2}+y^{2}+$ $z^{2}+t^{n}=0$ at $(0,0,0,0)$ by $\mathbb{Z} / 2 \mathbb{Z}$ acting via $\operatorname{diag}(-1,-1,-1,1)$. This time we restrict to $n \geq 2$, and do not include a smooth point in this family.

Each $P(n)$ is the quotient of a compound DuVal singularity by a group of order 2, and is terminal of index 2. A particularly simple resolution can be described inductively in the following way. There is a distinguished partial resolution $\tilde{\pi}: \widetilde{X} \rightarrow X$ whose exceptional divisor is $\overline{\mathbb{F}}_{4}$, such that $\widetilde{X}$ has a unique singular point (at the vertex of $\overline{\mathbb{F}}_{4}$ ) which has type $P(n-1)$ if $n \geq 3$, and type $Q(1)$ if $n=2$. (The "initial step" in this inductive resolution is the resolution of $Q(1)$ described earlier.)

3.4. Note. The resolutions of $Q(n), A(n)$ and $P(n)$ singularities occur naturally in the study of degenerations of surfaces with $\kappa=0$. In the notation of [CM1], the resolutions occur as flowers in flower-pot degeneration for the types of flowers shown in Table 5. The entries in that table were derived from Tables 3.2 and 3.4 of [CM1, pp. 365-367]; the notation $*$ indicates that we pass to the normal crossings resolution of $X$ by blowing up the singular point of the $\overline{\mathbb{F}}_{2}$ (a smooth point on $Y$ ). In all cases the pot meets the singularity normally as defined above. For $A(0)$, this means that $2 B$ corresponds to a smoothly contractible $\mathbb{P}^{2}$, the contraction of which would contract a -2 curve on the pot.

$Q(n), A(n)$, and $P(n)$ are all families of $\mathbb{Q}$-factorial terminal singularities for which there is a normal crossings resolution having no triple points (i.e. there are no triple points in the exceptional divisor). In a companion paper [CM2], we show that these are essentially all infinite families of such singularities, although there are also three "exceptional" cases. 
TABLE 6. Other flowers.

\begin{tabular}{|c|c|l|l|}
\hline Type Flower & $K_{Y}$ & $V_{0}$ & $V_{i}, i \geq 1$ \\
\hline $3 B$ & $0 V_{0}$ & $\mathbb{P}^{2}$ & - \\
$4 \alpha$ & $0 V_{0}$ & ruled & - \\
$4 C$ & $(l-2) V_{0}+(l-1) V_{1}+\cdots+1 V_{l-1}+0 V_{l}$ & $\mathbb{P}^{2}$ & $\mathbb{F}_{2}$ \\
$4 B$ & $-\frac{1}{4} V_{0}$ & $\mathbb{P}^{2}$ & - \\
$6 \alpha$ & $0 V_{0}+0 V_{1}$ & ruled & ruled \\
$6 C$ & $2(l-2) V_{0}+(l-3) V_{1}+\cdots+1 V_{l-2}+0 V_{l-1}+0 V_{l}$ & $\mathbb{P}^{2}$ & $\mathbb{F}_{2}, i<l ; F_{l} \cong \mathbb{F}_{4}$ \\
$6 E$ & $0 V_{0}$ & $\mathbb{P}^{2}$ & - \\
$6 \beta$ & $-\frac{1}{3} V_{0}$ & ruled & - \\
$6 D$ & $2(l-1) V_{0}+\frac{3 l-4}{2} V_{1}+\cdots+\frac{5}{3} V_{l-2}+\frac{2}{3} V_{l-1}-\frac{1}{3} V_{l}$ & $\mathbb{P}^{2}$ & $\mathbb{F}_{2}$ \\
$6 B$ & $-\frac{1}{2} V_{0}$ & $\mathbb{P}^{2}$ & - \\
$8 \alpha$ & $0 V_{0}+0 V_{1}+0 V_{2}$ & ruled & ruled \\
$8 \beta$ & $-\frac{1}{2} V_{0}$ & ruled & - \\
$12 \alpha$ & $0 V_{0}+0 V_{1}+0 V_{2}+0 V_{3}+0 V_{4}$ & ruled & ruled \\
$12 \beta$ & $-\frac{2}{3} V_{0}$ & ruled & - \\
\hline
\end{tabular}

3.5. Contraction of flowers. In additicn to the flowers of 3.4, there are other flowers which may be contracted to terminal singular points. To determine which may and which may not be so contracted, we compute $K_{Y}$ where $Y \rightarrow \Delta$ is a flower-pot degeneration of surfaces with $\kappa=0$, choosing the representative of $K_{Y}$ with $\operatorname{Supp} K_{Y} \subset Y_{0}$ and having multiplicity zero on the pot. This computation yields (for flowers other than those of Table 5) the results shown in Table 6.

In this table, the $V_{i}$ are the components of the flowers. Note that strictly positive coefficients correspond to terminal singularities, zero coefficients to canonical but not terminal singularities while negative coefficients correspond to noncanonical singularities. Those flowers and subflowers for which the coefficients of $K_{Y}$ are strictly positive may in fact be contracted to (terminal) points: it is an easy exercise to find explicit anti-ample divisors comprised of positive combinations of the $V_{i}$ 's and so the contraction exists by Grauert's criterion [G].

3.6. Proposition. If $Y \rightarrow \Delta$ is a flower-pot degeneration of surfaces with $\kappa=0$ with pot $P$ and $Y \rightarrow X$ contracts a flower to a terminal point $p \in X$, then either

1. $p \notin \sigma(P)$, or

2. $(X, p)$ is a singularity of type $Q(n), A(n)$ or $P(n)$.

Proof. By our table, if $\sigma^{-1}(p)$ is not of type $4 D$, then the coefficient of $V_{l}$ in $K_{Y}$ is less than or equal to zero, so $V_{l}$ (which is the component of the flower meeting the pot) will not be contracted by $\sigma$.

3.7. Proposition. If $Y \rightarrow \Delta$ is a flower-pot degeneration of surfaces with $\kappa=0$ and $\sigma: Y \rightarrow X$ contracts all terminal flowers and subflowers of $Y$, then either

1. $K_{X} \equiv 0$, or

2. $Y$ has flowers of types $4 B, 6 B, 6 D, 6 \beta, 8 \beta$, or $12 \beta$.

Proof. Only the flowers mentioned in 2 have negative coefficients in $K_{Y}$. 
3.8. Proposition. There is a birational model $Z$ of $Y$ obtained by contracting all flowers to points. The singularities of $Z$ are log-terminal, but may be worse than canonical.

Proof. The existence of the model follows easily from Grauert's contraction criterion $[G]$. Since the coefficients of the exceptional divisors in the canonical bundle are all greater than -1 , the singularities are log-terminal. But since some coefficients may indeed be negative, the singularities are not always canonical.

\section{ELEMENTARY MODIFICATIONS}

We construct in this section the elementary modifications (or flips) needed for the proof of our main theorem. Our construction generalizes the classical construction of flips centered on smooth rational curves $C$ with normal bundles $\mathscr{O}(-1) \oplus \mathscr{O}(-1)$ or $\mathscr{O} \oplus \mathscr{O}(-2)$, as described in [R1] or [P] (among other places). Recall that in the case of normal bundle $\mathscr{O}(-1) \oplus \mathscr{O}(-1)$, one simply blows up the curve to obtain an exceptional divisor isomorphic to $\mathbb{P}^{1} \times \mathbb{P}^{1}$, and then contracts that exceptional divisor along its other ruling to a curve $C^{\prime}$. This is a symmetric flip, which means that neighborhoods of $C$ and $C^{\prime}$ have identical numerical properties. We first construct some symmetric flips, and then use them to construct the asymmetric flips which are needed for the proof of our main theorem.

We begin by analyzing normal bundles of smooth rational curves in smooth threefolds, using methods similar to [Mrr2, Theorem 3.2].

4.1. Lemma. Let $C$ be a complete smooth rational curve contained in $S$ and $T$, surfaces smooth near $C$, with $S$ and $T$ contained in $X$, a threefold smooth near $C$. Assume $S$ and $T$ meet transversely except at points $P_{1}, \ldots, P_{\alpha}$ of $C$ at each of which, in appropriate local coordinates $u, v$ and $w$ on $X, C=$ $\{(u, v, w) \mid u=v=0\}, S=\{(u, v, w) \mid u=v w\}$ and $T=\{(u, v, w) \mid u=$ $0\}$. If $d=\left(C^{2}\right)_{S}$ and $e=\left(C^{2}\right)_{T}$, then

1. $K_{X} \cdot C=-d-e-\alpha-2$,

2. if $d-e \geq \alpha$, then $N_{C / X} \cong \mathscr{O}_{C}(d) \oplus \mathscr{O}_{C}(e+\alpha)$, and

3. if $\alpha>d-e \geq 0$, then there is an integer $n$ such that $0 \leq n \leq(\alpha-d+e) / 2$, and

$$
N_{C / X} \cong \mathscr{O}_{C}(d+n) \oplus \mathscr{O}_{C}(e+\alpha-n) .
$$

Proof. Let $\rho_{S}: N_{C / X}^{*} \rightarrow N_{C / S}^{*}$ and $\rho_{T}: N_{C / X}^{*} \rightarrow N_{C / T}^{*}$ be the natural maps on conormal sheaves induced by restriction. $S, T$ and $X$ are smooth near $C$, so these maps are surjective with kernels $N_{S / X}^{*} \otimes \mathscr{O}_{C}$ and $N_{T / X}^{*} \otimes \mathscr{O}_{C}$, respectively. Consider $\rho=\Lambda^{2}\left(\rho_{S} \oplus \rho_{T}\right)$, so

$$
\rho: \bigwedge^{2} N_{C / X}^{*} \rightarrow \bigwedge^{2}\left(N_{C / S}^{*} \oplus N_{C / T}^{*}\right) .
$$

Near a point where $S$ and $T$ meet transversely, $N_{C / X} \cong N_{C / S} \oplus N_{C / T}$, so at such points, $\rho$ is an isomorphism. Near $P_{i}, 1 \leq i \leq \alpha, \Omega_{X} \otimes \mathscr{O}_{C}$ is generated by $d u, d v$ and $d w$ as $\mathscr{O}_{C}$-module. Since $N_{C / X}^{*}=\operatorname{Ker}\left(\Omega_{X} \otimes \mathscr{O}_{C} \rightarrow \Omega_{C}\right)$, $N_{C / X}^{*}$ is generated by $d u$ and $d v$. Similarly, $N_{C / S}^{*}$ is generated by $d u$ and $d v$ with relation $d u=w d v$ and $N_{C / T}^{*}$ is generated by $d u$ and $d v$ with relation $d u=0$. Thus 


$$
\rho_{S}(f d u+g d v)=(w f+g) \rho_{S}(d v) \quad \text { and } \quad \rho_{T}(f d u+g d v)=g \rho_{T}(d v)
$$

and so $\rho(d u \wedge d v)=w \rho_{S}(d v) \wedge\left(\rho_{S}(d v)+\rho_{T}(d v)\right)=w \rho_{S}(d v) \wedge \rho_{T}(d v)$. Now $\rho_{S}(d v) \wedge \rho_{T}(d v) \neq 0$ and $w$ is a local equation of $P_{i}$ on $C$, so $\rho: \wedge^{2} N_{C / X}^{*} \stackrel{\sim}{\rightarrow}$ $\Lambda^{2}\left(N_{C / S}^{*} \oplus N_{C / T}^{*}\right) \otimes I_{Z}$ where $Z=P_{1}+\cdots+P_{\alpha}$. Thus $\operatorname{deg} \bigwedge^{2} N_{C / X}^{*}=-d-e-\alpha$ and so, by adjunction, since $C$ is rational,

$$
K_{X} \cdot C=-\operatorname{deg} \bigwedge^{2} N_{C / X}-2=-d-e-\alpha-2
$$

giving 4.1.1.

To show 4.1.2 and 4.1.3, we may assume that $d \geq e$ and $N_{C / X} \cong \mathscr{O}_{C}(a) \oplus$ $\mathscr{O}_{C}(b)$ where $a \geq b$. Thus we have $a+b=d+e+\alpha$ since $\operatorname{deg} \bigwedge^{2} N_{C / X}=$ $-d-e-\alpha$; moreover, we have a surjective homomorphism $\rho_{S}: \mathscr{O}_{C}(-a) \oplus$ $\mathscr{O}_{C}(-b) \rightarrow \mathscr{O}_{C}(-d)$ which therefore corresponds to a nowhere vanishing element of $H^{0}\left(C, \mathscr{O}_{C}(a-d) \oplus \mathscr{O}_{C}(b-d)\right)$, so either $h^{0}\left(C, \mathscr{O}_{C}(a-d) \oplus \mathscr{O}_{C}(b-d)\right)$ is one or is strictly greater than $h^{0}\left(C, \mathscr{O}_{C}(a-d)\right)$, since $a-d \geq b-d$. Thus either $b-d \geq 0$ or $a-d=0$.

If $b-d \geq 0$, let $n=b-d$. Now $a=d+e+\alpha-b=e+\alpha-n$ and $b=d+n$ as required for $N_{C / X}$ in case 4.1.3. By assumption $a \geq b$ so $e+\alpha-n \geq d+n$. Thus $n \leq(\alpha-d+e) / 2$ and $\alpha-(d-e) \geq 2 n \geq 0$. If $\alpha>d-e$, we are in case 4.1.3. If $\alpha=d-e$, then $n=0$ and 4.1.2 and 4.1.3 both hold.

If $a-d=0$, then $a=d$ and $b=d+e+\alpha-a=e+a$. Now $a \geq b$ so $d \geq e+\alpha$ and $d-e \geq \alpha$. Thus if $d-e>\alpha$, then $a-d=0$ and so 4.1.2 holds; if $d-e=\alpha$ then 4.1.2 holds (as does 4.1.3 with $n=0$ ) and if $\alpha>d-e \geq 0$ then $b-d \geq 0$ and 4.1.3 holds with $n=b-d$.

4.2. Lemma. Let $C$ be a smooth rational curve contained in a surface $S$ contained in a threefold $X$ smooth near $C$. Assume $S$ is smooth near $C$ except at $P_{1}, \ldots, P_{\alpha}$ on $C$ where $S$ has ordinary double points. Let $\delta=\left(C^{2}\right)_{S}$ and $m=K_{X} \cdot C$.

Let $\pi: \widetilde{X} \rightarrow X$ blow up $C, \widetilde{S}$ be the proper transform of $S$ in $\tilde{X}, \tilde{C}$ be the proper transform of $C$ in $\widetilde{S}$ and $E=\pi^{-1}(C)$. Also let $\widetilde{P}_{1}, \ldots, \widetilde{P}_{\alpha}$ be the points on $\widetilde{C}$ lying over $P_{1}, \ldots, P_{\alpha}$ on $C$. Then

1. $\widetilde{C} \subset \widetilde{S} \cap E \subset \widetilde{X}$ satisfies the conditions of Lemma 4.1 with nontransverse points $\widetilde{P}_{1}, \ldots, \widetilde{P}_{\alpha}$.

2. $\left(\widetilde{C}^{2}\right)_{\tilde{S}}=\delta-\frac{1}{2} \alpha$ and $\left(\widetilde{C}^{2}\right)_{E}=-2 \delta-m-\alpha-2$, and Then

3. Let $N_{C \mid X}=\mathscr{O}_{C}(a) \oplus \mathscr{O}_{C}(b)$ with $a \geq b$, so $b=-a-m-2, E \cong \mathbb{F}_{a-b}$.

(i) $0 \leq 2 a+m+2 \leq\left(\widetilde{C}^{2}\right)_{E}$ or

(ii) $\left(\widetilde{\widetilde{C}}^{2}\right)_{E} \leq 0$ and $2 a+m+2=-\left(\widetilde{C}^{2}\right)_{E}$ i.e. $a=\delta+\frac{1}{2} \alpha$ (by 4.2.2).

Proof. $\tilde{S}$ and $E$ meet normally except at $P_{i}, 1 \leq i \leq \alpha$. In local coordinates $x, y, z$ near $P_{i}$ we may write $C=\{(x, y z) \mid x=y=0\}$ and $S=\left\{(x, y, z) \mid x^{2}=y z\right\}$. On the blow-up of $X$, we have the coordinate chart $u=x, v=y / x$ and $w=z$ in which $\widetilde{S}=\{(u, v, w) \mid u=v w\}$ and $E=\{(u, v, w) \mid u=0\}$, so $\widetilde{C} \subset \widetilde{S} \cap E \subset \widetilde{X}$ satisfies the conditions of Lemma 4.1. 
Let $d=\left(\widetilde{C}^{2}\right)_{\tilde{S}}$ and $e=\left(\widetilde{C}^{2}\right)_{E}$. Let $\sigma=\left.\pi\right|_{\tilde{S}}: \widetilde{S} \rightarrow S$, so $\sigma^{*} C \equiv \widetilde{C}+$ $\frac{1}{2}\left(\sigma^{-1}\left(P_{1}\right)+\cdots+\sigma^{-1}\left(P_{\alpha}\right)\right)$ and so $\left(C^{2}\right)_{S}=\left(\widetilde{C}^{2}\right)_{\tilde{S}}+\frac{1}{4}(-2 \alpha)+2 \cdot \frac{1}{2} \alpha$ since $\widetilde{C} \cdot \sigma^{-1}\left(P_{i}\right)=1$ for all $i$ and $\left(\sigma^{-1}\left(P_{i}\right)\right)_{\widetilde{S}}^{2}=-2$. Thus $d=\delta-\frac{1}{2} \alpha$ as claimed in 4.2.2. To compute $e$, we use adjunction applied to $\widetilde{C}$ on $E$ to get $K_{E} \cdot \widetilde{C}=$ $-2-e$ (since $\widetilde{C}$ is rational). Now $K_{E} \equiv K_{\widetilde{X}} \cdot E+E \cdot E$ and $K_{\widetilde{X}} \equiv \pi^{*} K_{X}+E$ since $\pi$ is the blow-up of a curve. Now $\pi_{*} \pi^{*} K_{X} \cdot E \equiv K_{X} \cdot \mathscr{C}^{\prime}=m$ (point on $C$ ), so $\pi^{*} K_{X} \cdot E \equiv m F$ for $F$ a fiber on $E$. Thus $K_{E} \equiv m F+2 E \cdot E$. Intersecting with $\widetilde{C}$ gives $-2-e=m+2((E \cdot E) \cdot \widetilde{C})_{E}$ since $\widetilde{C} \cdot F=1$. Now $((E \cdot E) \cdot \widetilde{C})_{E}=(E \cdot \widetilde{C})_{\tilde{X}}$ so $E \cdot \widetilde{C}=-1-\frac{1}{2} e-\frac{1}{2} m$. On the other hand, $E \cdot \widetilde{C}=K_{\widetilde{X}} \cdot \widetilde{C}-\pi^{*} K_{X} \cdot \widetilde{C}$. By Lemma 4.1.1, E $\cdot \widetilde{C}=-d-e-\alpha-2-m$. Combining these gives $e=-2 d-2 \alpha-2-m$. Using $d=\delta-\frac{1}{2} \alpha$ gives the desired value for $e$, proving 4.2.2.

For 4.2.3, note that $b=-a-m-2$ follows from adjunction applied to $C$ in $X$. That $E \cong \mathbb{F}_{a-b}$ is clear since $E=\mathbb{P}\left(N_{C / X}\right)$. $\widetilde{C}$ is a section on $\mathbb{F}_{a-b}$ so either $\left(\widetilde{C}^{2}\right)_{E}=a-b+2 k, k \geq 0$, or $\left(\widetilde{C}^{2}\right)_{E}=b-a$ since any section other than the negative section is linearly equivalent on $E$ to $S_{0}+k F$ for $\left(S_{0}^{2}\right)_{E}=a-b$, $F$ a fiber and $k \geq 0$. If $\left(\widetilde{C}^{2}\right)_{E}=a-b+2 k$, then using $b=-a-m-2$, we have $(\widetilde{C})_{E}=a-b+2 k \geq a-b=2 a+m+2 \geq 0$ since $a-b \geq 0$, giving 4.2.3i. If $\left(\widetilde{C}^{2}\right)_{E}=b-a$, then $\left(\widetilde{C}^{2}\right)_{E} \leq 0$ and $-\left(\widetilde{C}^{2}\right)_{E}=a-b=2 a+m+2$ giving 4.2.3(ii).

We can now construct our symmetric flips.

4.3. Proposition. Let $C$ be a complete smooth rational curve such that $C \subset S \subset$ $X, S$ a surface and $X$ a threefold. Assume $P \in C$ is a singular point of $X$ of type $A(m), m \geq 0, C$ and $S$ meet $P$ normally (as defined in $\S 3$ ) and $S$ and $X$ are otherwise smooth near $C$. If $\left(C^{2}\right)_{S}=-\frac{1}{2}$ and $K_{X} \cdot C=0$, then there is a birational modification $f: X \rightarrow \bar{X}$ such that

1. $f: X-C \stackrel{\sim}{\rightarrow} \bar{X}-\bar{C}$ for some complete smooth rational curve $\bar{C}$.

2. $\bar{X}$ is smooth near $\bar{C}$ except for a singularity of type $A(m)$ at $\bar{P} \in \bar{C}$.

3. For $\bar{S}$ the proper transform of $S$ under $f$, the induced rational map $\left.f\right|_{S}: S \rightarrow \bar{S}$ blows down $C, \bar{S}$ is smooth near $\bar{C}, \bar{P} \notin \bar{C}$ and $\bar{S}$ meets $\bar{C}$ transversely.

4. If $T$ is a surface in $X$ transverse to $C$ and $S$ such that $P \notin T$ and $T$ is smooth near $C \cap T$, then $\bar{T}$, the proper transform of $T$, and $\bar{C}$ meet $\bar{P}$ normally, $\bar{T}$ is otherwise smooth near $\bar{C},\left(\bar{C}^{2}\right)_{\bar{T}}=-\frac{1}{2}$ and $K_{\bar{X}} \cdot \bar{C}=0$ i.e., $f$ is a symmetric modification.

Proof. We use induction on $m$. Suppose first that $m=0$. Note that $X$ is smooth in this case. Let $\pi: \widetilde{X} \rightarrow X$ blow up $C$. Using the notation and results of Lemma 4.2 , we have $\alpha=1, \delta=-\frac{1}{2}$ and $m=0$, so $\left(\widetilde{C}^{2}\right)_{\widetilde{S}}=-1,\left(\widetilde{C}^{2}\right)_{E}=$ $-2, N_{C / X} \cong \mathscr{O}_{C} \oplus \mathscr{O}_{C}(-2)$ and $E \cong \mathbb{F}_{2}$. Applying Lemma 4.1 to $\tilde{C} \subset \tilde{S} \cap E$ with $d=-1, e=-2$ and $\alpha=1$ yields, by 4.1.2, $N_{\widetilde{C} / \widetilde{X}} \cong \mathscr{O}_{\widetilde{C}}(-1) \oplus \mathscr{O}_{\widetilde{C}}(-1)$ and $K_{\widetilde{X}} \cdot \widetilde{C}=0$. Let $\beta: \widetilde{X} \rightarrow X^{\prime}$ be the symmetric flip centered at $\widetilde{C}$, so $\beta$ blows down $\tilde{C}$ on $\tilde{S}$. Lemma 4.2 shows that the proper transform of $E$ intersects the blow-up of $\widetilde{C}$ (isomorphic to $\mathbb{P}^{1} \times \mathbb{P}^{1}$ ) along a diagonal and so $\beta$ 

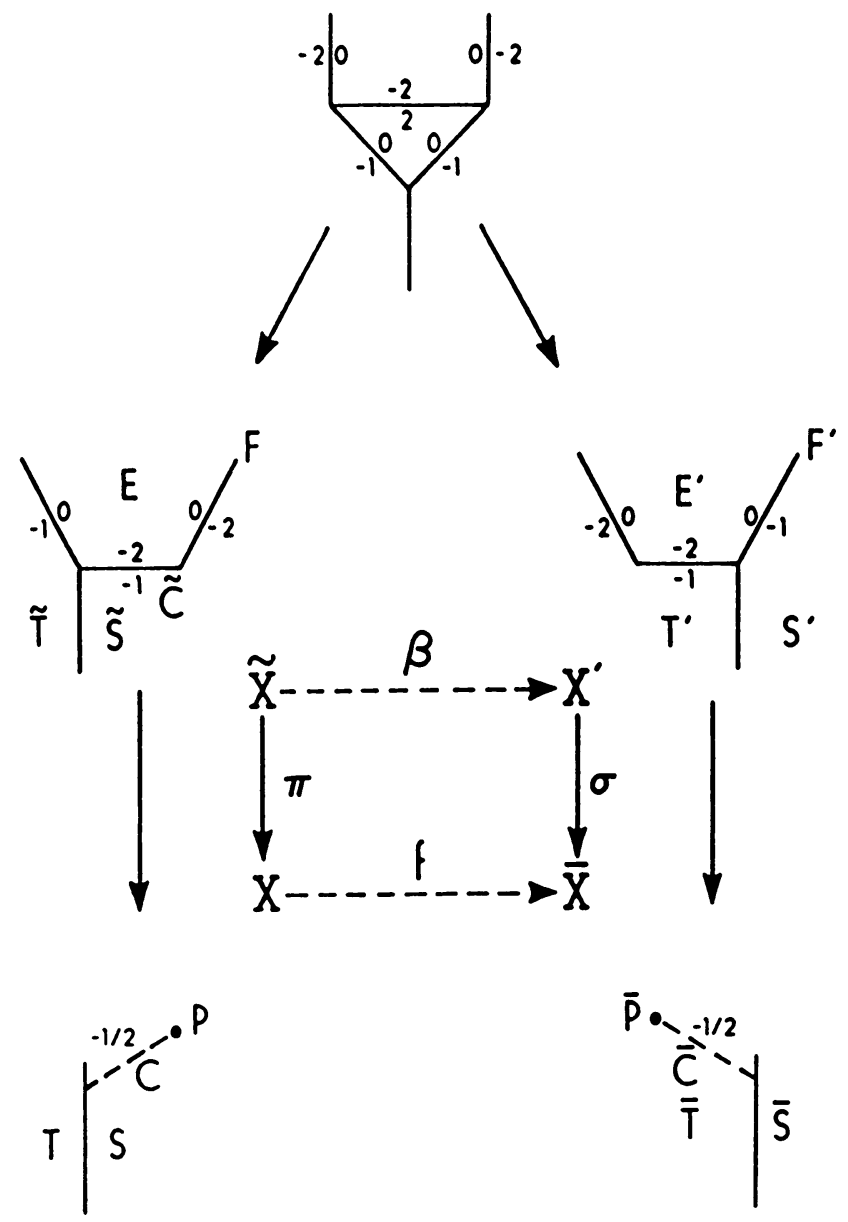

Figure 2. Construction of $f$ when $m=0$.

maps $E$ isomorphically to its proper transform $E^{\prime} \cong \mathbb{F}_{2}$. Now $E \cap \widetilde{S}=\widetilde{C} \cup F$ for $F$ a fiber on $E$; moreover, $\left(F^{2}\right)_{\tilde{S}}=-2$ since $F$ is the exceptional curve of the resolution of the double point on $S . F \cdot \widetilde{C}=1$, so when $\widetilde{C}$ is blown down by $\beta$, we have $F^{\prime}=E^{\prime} \cap S^{\prime}, F^{\prime}$ still a fiber on $E^{\prime}$ and $\left(F^{2}\right)_{S^{\prime}}=-1$, so $E^{\prime}$ is smoothly contractible in $X^{\prime}$. Letting $\sigma: X^{\prime} \rightarrow X$ blow down $E^{\prime}$ to a curve $\bar{C}$ and $f=\sigma \circ f \circ \pi^{-1}, 4.3 .1-4.3 .3$ are verified. Property 4.3.4 is easily verified. Figure 2 illustrates our procedure, including the blow-up and blow-down which constitute $\beta$.

Now suppose that the proposition is true for $m-1$; we will prove it for $m$. Let $\pi_{1}: X_{1} \rightarrow X$ be the distinguished partial resolution of $P$. Let $E$ be $\pi_{1}^{-1}(P)$, so $E \cong \overline{\mathbb{F}}_{2}$, and let $\Delta=E \cap S_{1}$ (subscripts indicate proper transforms). By the assumption that $C$ and $S$ meet $P$ normally, $C_{1}$ meets $E$ normally, $\Delta \cdot \Gamma=1$ for a generator $\Gamma$ on $E,\left(\Delta^{2}\right)_{E}=2$ and $P_{1}$, the singular point of $X_{1}$, lies on $E-\Delta$. By Lemma 4.2, $\left(C_{1}^{2}\right)_{S_{1}}=-1$ and, since $\pi_{1}$ resolves the double point on $S,\left(\Delta^{2}\right)_{S_{1}}=-2$. Note that $K_{X_{1}} \equiv \pi_{1}^{*} K_{X}+E$. Thus $K_{X_{1}} \cdot C_{1}=\pi_{1}^{*} K_{X} \cdot C_{1}+E \cdot C_{1}=K_{X} \cdot C+E \cdot C_{1}=0+1=1$. Let $\Gamma$ be the generator on the $\overline{\mathbb{F}}_{2}$ passing through $C_{1} \cap E$. By $0.7,\left(\Gamma^{2}\right)_{E}=\frac{1}{2}$ and 


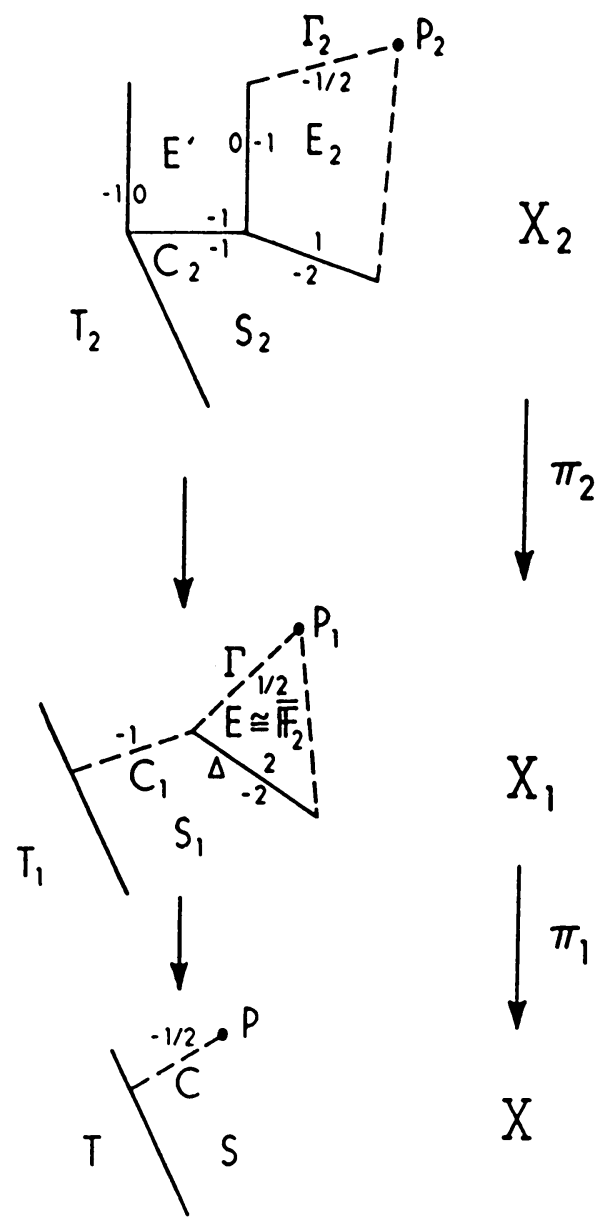

Figure 3. Partial construction (induction step).

$K_{E} \equiv-4 \Gamma$. Now $K_{E}=\left(\pi^{*} K_{X}+E\right) \cdot E+E \cdot E=2 E \cdot E$ so $E \cdot E \equiv-2 \Gamma$ on $E$. Thus

$$
\begin{aligned}
K_{X_{1}} \cdot \Gamma & =\pi^{*} K_{X} \cdot \Gamma+E \cdot \Gamma=E \cdot \Gamma=((E \cdot E) \cdot \Gamma)_{E} \\
& =-2\left(\Gamma^{2}\right)_{E}=-2 \cdot \frac{1}{2}=-1 .
\end{aligned}
$$

Let $\pi_{2}: X_{2} \rightarrow X_{1}$ blow up $C_{1}$ with exceptional divisor $E^{\prime}$. Let $C_{2}=E^{\prime} \cap S_{2}$. By Lemma 4.2 applied to $C_{1} \subset S_{1}$, so $\delta=-1, \alpha=0$ and $m=1$, we have $\left(C_{2}^{2}\right)_{S_{2}}=-1$ and $\left(C_{2}^{2}\right)_{E^{\prime}}=-1$ so $E^{\prime} \cong \mathbb{F}_{1}$ and $N_{C_{2} / X_{2}} \cong \mathscr{O}_{C_{2}}(-1) \oplus \mathscr{O}_{C_{2}}(-1)$. Figure 3 illustrates the procedure so far.

To finish, we will use the inductive hypotheses to flip $\Gamma_{2}$ and $P_{2}$ onto $E^{\prime}$, perform an elementary modification at $C_{2}$, and blow down $E^{\prime}$. Now $\left(\Gamma_{2}^{2}\right)_{E_{2}}=$ $-\frac{1}{2}$ and $K_{X_{2}}=\pi_{2}^{*} K_{X_{1}}+E^{\prime}$ (since $\pi_{2}$ is a smooth blow-up of a curve), so

$$
K_{X_{2}} \cdot \Gamma_{2}=\pi_{2}^{*} K_{X_{1}} \cdot \Gamma_{2}+E^{\prime} \cdot \Gamma_{2}=K_{X_{1}} \cdot \Gamma+1=-1+1=0
$$

so $\Gamma_{2}$ satisfies the inductive hypotheses and so there is a modification $\gamma: X_{2} \rightarrow \rightarrow$ $X_{3}$ such that $\gamma: X_{2}-\Gamma_{2} \stackrel{\sim}{\rightarrow} X_{3}-\Gamma_{3}, \gamma$ blows down $\Gamma_{2}$ on $E_{2}$ and, since $E^{\prime}$ is transverse to $\Gamma_{2}, E^{\prime}$ is transformed as in 4.3.4. All this has left $C_{2}$ (now called 


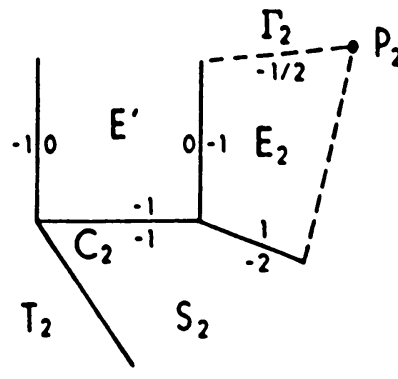

$\mathrm{X}_{2}$

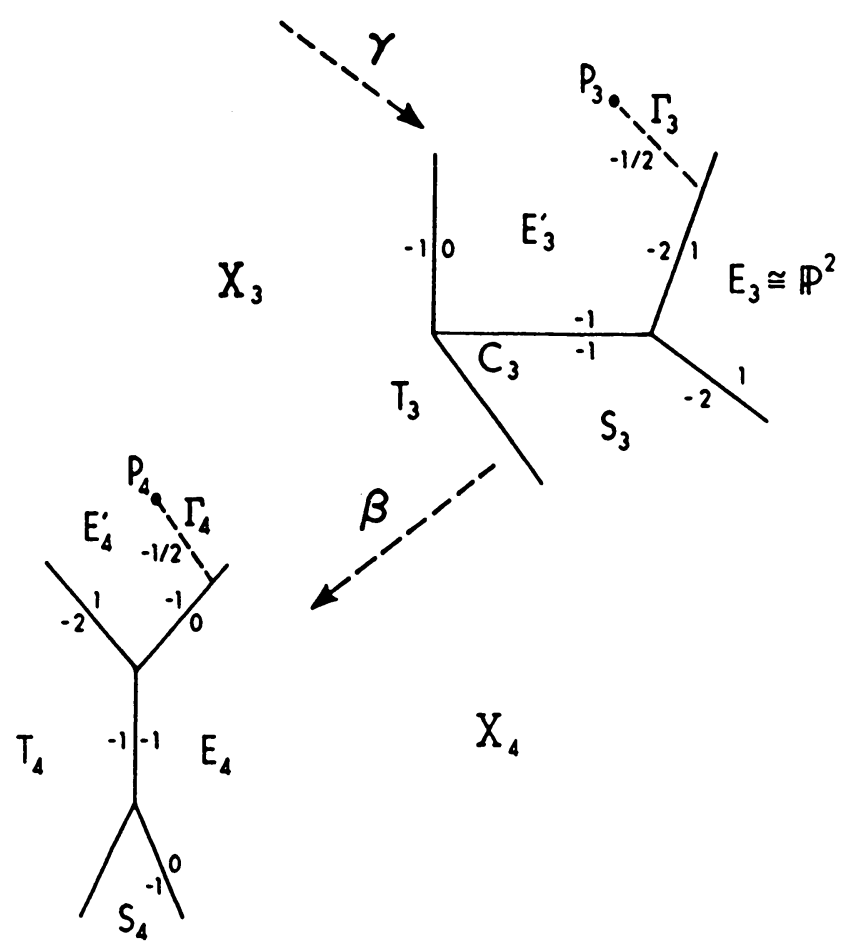

FIGURE 4. Further construction (induction step)

$C_{3}$ ) unaffected so let $\beta: X_{3} \rightarrow X_{4}$ be an elementary modification centered at $C_{3}$. Figure 4 illustrates the effect of $\gamma$ and $\beta$.

Now $E_{4} \cong \mathbb{F}_{1}$ and is smoothly contractable. Let $\sigma_{1}: X_{4} \rightarrow X_{5}$ blow down $E_{4}$. Now $E_{5}^{\prime} \cong \overline{\mathbb{F}}_{2}$ with an $A(m-1)$ singularity at the vertex $P_{5}$ and $T_{5} \cap E_{5}^{\prime}$ is a section on $E_{5}^{\prime}, P_{5} \notin T_{5} \cap E_{5}^{\prime}$. The normal bundle of $E_{5}^{\prime}$ is negative, and when $E_{5}^{\prime}$ is blown down by $\sigma_{2}: X_{5} \rightarrow X_{6}$, the image of $E_{5}^{\prime}$ is an $A(m)$ singular point $P_{6}$ on $T_{6}$ away from $S_{6}$. Letting $f=\sigma_{2} \circ \sigma_{1} \circ \beta \circ \gamma \circ \pi_{2}^{-1} \circ \pi_{1}^{-1}$ proves the proposition.

4.4. Remark. Proposition 4.3 with $m=0$ can be used to characterize rational curves $C$ in smooth threefolds $X$ with the following property: $N_{C / X}=\mathscr{O} \oplus$ $\mathscr{O}(-2)$, and if we let $\widetilde{C}=\sigma_{\infty}$ be the curve of negative self-intersection in the exceptional divisor $\mathbb{F}_{2}$ of the blowup $\tilde{X} \rightarrow X$ of $C$, then $N_{\widetilde{C} / \widetilde{X}}=\mathscr{O}(-1) \oplus$ $\mathscr{O}(-1)$. (In the terminology of Pinkham [P, Theorem 4], $C$ has normal bundle sequence $\mathscr{O} \oplus \mathscr{O}(-2), \mathscr{O}(-1) \oplus \mathscr{O}(-1)$.) The characterization is this: $C$ has 
such a normal bundle sequence if and only if there is a surface $S$ containing $C$ which has a single ordinary double point at some point of $C$ (and is otherwise smooth) such that $\left(C^{2}\right)_{S}=-\frac{1}{2}$ and $K_{X} \cdot C=0$. That this condition is sufficient follows from the proof of 4.3. To see the necessity, note that the sequence of blow-ups and blow-downs used in the proof can be applied to any curve with that normal bundle sequence, to yield $\bar{C} \subset \bar{X}$. If we take $\bar{S} \subset \bar{X}$ transverse to $\bar{C}$ and invert the modification, the proper transform of $\bar{S}$ is the desired surface $S$ containing $C$.

Another application of Proposition 4.3 is the construction of certain contractions of ruled surfaces with singularities.

4.5. Corollary. Let $S$ be a generically contractible surface in a threefold $X$ where $\varphi: S \rightarrow \Gamma$ gives $S$ the structure of ruled surface. Assume that

1. The only singular fibers of $\varphi$ are $F_{1}, \ldots, F_{\alpha}$ where $F_{i} \equiv 2 C_{i}, C_{i}$ smooth rational curves, $S$ has ordinary double points at $P_{i}$ and $Q_{i}$ which are contained in $C_{i}$ and $S$ and $C_{i}$ meet $P_{i}$ and $Q_{i}$ normally,

2. $S-\left\{P_{1}, \ldots, P_{\alpha}, Q_{1}, \ldots, Q_{\alpha}\right\}$ and $X-\left\{P_{1}, \ldots, P_{\alpha}, Q_{1}, \ldots, Q_{\alpha}\right\}$ are smooth, and

3. $X$ has singularities of type $A\left(k_{i}\right)$ at $P_{i}$ and type $Q(1)$ at $Q_{i}$ for all $i=1, \ldots, \alpha$.

Then there exists a contraction $\sigma: X \rightarrow Y$ such that $\sigma(S)=\Gamma,\left.\sigma\right|_{S}=\varphi$, and $Y$ is smooth except at $R_{1}, \ldots, R_{\alpha}$, where $R_{i}=\sigma\left(F_{i}\right)$, at which $Y$ has singularities of types $A\left(k_{i}+1\right)$.

Proof. Note that since $S$ is generically contractible, for $F$ a general fiber of $\varphi,-1=S \cdot F=\operatorname{deg}_{F}\left(\left.N_{S / X}\right|_{F}\right)$. Now

$$
\left.0 \rightarrow N_{F / S} \rightarrow N_{F / X} \rightarrow N_{S / X}\right|_{F} \rightarrow 0
$$

and $\operatorname{deg}_{F} N_{F / S}=0$, so $\operatorname{deg}_{F} N_{F / X}=-1$. By adjunction, therefore, $K_{x} \cdot F=-1$ and so, by $4.5 .1, K_{X} \cdot C_{i}=-\frac{1}{2}$ for $i=1, \ldots, \alpha$.

Let $\tau: \widetilde{X} \rightarrow X$ be the blow-up of $Q_{1}, \ldots, Q_{\alpha}$. Letting $E_{i}=\tau^{-1}\left(Q_{i}\right)$ and $\sim$ denote proper transform, $\widetilde{S}$ is smooth except for ordinary double points at $P_{1}, \ldots, P_{\alpha}, E_{i} \equiv \mathbb{P}^{2}$ and $K_{\widetilde{X}} \equiv \tau^{*} K_{X}+\frac{1}{2}\left(E_{1}+\cdots+E_{\alpha}\right)$. Thus $K_{\widetilde{X}} \cdot \widetilde{C}_{i}=$ $-\frac{1}{2}+\frac{1}{2}=0$ since $C_{i}$ meets $Q_{i}$ normally; moreover, by $0.6,\left(\widetilde{C}_{i}^{2}\right)_{\widetilde{S}}=\left(C_{i}^{2}\right)_{S}-\frac{1}{2}=$ $-\frac{1}{2}$, so $\widetilde{C}_{i} \subset \widetilde{S} \subset \widetilde{X}$ satisfy the conditions of Proposition 4.3. Applying the modification to each of $\widetilde{C}_{1}, \ldots, \widetilde{C}_{\alpha}$ we obtain $\bar{X}$ on which $\bar{S}$, the proper transform of $\widetilde{S}$, is smooth, by 4.3 .3 , and minimal ruled. Thus $\bar{S}$ is smoothly contractable to $\bar{\Gamma} \cong \Gamma$ in $\bar{X}$. Let $\bar{X} \rightarrow X^{\prime}$ be that contraction and $E_{i}^{\prime}$ the proper transform of $E_{i}$. The contraction of $\widetilde{S}$ blows down the exceptional curve $\bar{S} \cap \bar{E}_{i}$, yielding $E_{i}^{\prime} \cong \overline{\mathbb{F}}_{2}$ with the $A\left(k_{i}\right)$ singularities $\bar{Q}_{i}$ at the vertices of the $\overline{\mathbb{F}}_{2}$. Thus the $E_{i}^{\prime}$ 's may be contracted to $A\left(k_{i}+1\right)$ singularities on a threefold $Y$. The induced rational map $\sigma: X \rightarrow Y$ is in fact holomorphic, and gives the desired contraction.

4.6. Proposition. Let $C$ be a complete smooth rational curve such that $C \subset S \subset$ $X, S$ a surface, $X$ a threefold, $P \in C$ a singular point of $X$ of type $Q(n)$, $Q \in C$ either a smooth point of $X$, or a singular point of $X$ of type $A(m)$, $C$ and $S$ meet $P$ and $Q$ normally and $S$ and $X$ are otherwise smooth near $C$. If either 
A. $n \geq 1, Q$ is a smooth point of $X,\left(C^{2}\right)_{S}=-\frac{n}{n+1}$ and $K_{X} \cdot C=-\frac{1}{n+1}$ or

B. $n \geq 2, Q$ has type $A(m)$ for some $m \geq 0 \quad\left(C^{2}\right)_{S}=\frac{1}{2}-\frac{n}{n+1}$ and $K_{X} \cdot C=-\frac{1}{n+1}$, then there is a birational modification $f: X \rightarrow \frac{2}{X}$ such that

1. $f: X-C \stackrel{\sim}{\rightarrow} \bar{X}-\bar{C}$, for some complete smooth rational curve $\bar{C}$,

2. $\bar{X}$ is smooth near $\bar{C}$ except, in case $\mathrm{A}$, for a singularity of type $Q(n-1)$ at $\bar{P} \in \bar{C}$, and, in case $\mathrm{B}$, for singularities of type $Q(n-2)$ at $\bar{P}$ and type $A(m+1)$ at $\bar{Q}$ where $\bar{P}, \bar{Q} \in \bar{C}$, and

3. For $\bar{S}$ the proper transform of $S$ under $f,\left.f\right|_{S}: S \rightarrow \bar{S}$ blows down $C$, leaving $\bar{S}$ with a quotient singularity locally analytically isomorphic to $\mathbb{A}^{2}$ modulo $(x, y) \mapsto(\zeta x, \zeta y)$ where, in case $\mathrm{A}$, $\zeta$ is a primitive nth root of unity and, in case $\mathrm{B}, \zeta$ is a primitive $(n-1)$ st root of unity.

4.7. Remarks. 1. The modifications in case A were used by Danilov [D] and Reid [R2] to find relative (numerical) minimal models for projective toric morphisms. They are described for example, in [R2, Example (3.8), (in which one should set $a=0)$ ].

2. The contraction constructed in Corollary 4.5 corresponds to the "missing" case $n=1$ in case $\mathrm{B}$.

Proof of Proposition 4.6. The modification $f$ differs in details only between cases A and B. In both cases, our proof proceeds by induction on $n$. The relation between cases A and B may be explained by noting that whenever an elementary modification is used in case $\mathrm{A}$, there is an analogous curve to which the modification of Proposition 4.3 may be applied in case B.

In all cases, let $\sigma: \widetilde{X} \rightarrow X$ be the distinguished partial resolution of $P$. From the definitions of $\S 3, K_{\widetilde{X}} \equiv \sigma^{*} K_{X}+\frac{1}{n+1} V$ and $\sigma^{*} S \equiv \widetilde{S}+\frac{n}{n+1} V$. Thus

$$
K_{\widetilde{X}} \cdot \widetilde{C}=\pi^{*} K_{X} \cdot \widetilde{C}+\frac{1}{n+1} V \cdot \widetilde{C}=-\frac{1}{n+1}+\frac{1}{n+1}=0
$$

and by 0.6 ,

$$
\left(\widetilde{C}^{2}\right)_{\widetilde{S}}=\left(C^{2}\right)_{S}-\frac{1}{n+1}= \begin{cases}-1 & \text { in case A, } \\ -1 / 2 & \text { in case B. }\end{cases}
$$

Thus in case A, by 4.2.3, $N_{\widetilde{C} / \widetilde{X}} \cong \mathscr{O}_{\widetilde{C}}(-1) \oplus \mathscr{O}_{\widetilde{C}}(-1)$ and so an elementary modification may be performed center at $C$, while in case B, $\widetilde{C} \subset \widetilde{S} \subset \tilde{X}$ satisfies the hypotheses of Proposition 4.3. Let $\widetilde{X} \rightarrow \widehat{X}$ be the modification centered at $\widetilde{C}$ which, in case A, is an elementary modification and, in case B, is that of Proposition 4.3. Let - denote proper transform.

Let $F \subset V \subset \widetilde{X}$ be a generator on $V$ (under $\left.\left.\sigma\right|_{V}\right)$. Now $\left(F^{2}\right)_{V}=\frac{1}{n}$, $\left(E^{2}\right)_{V}=n$ and rank Pic $V=1$, so, on $V, F \equiv \frac{1}{n} E$. Thus, since $V \cdot E=$ $\left(E^{2}\right)_{\widetilde{S}}=-n-1$, we have

$$
K_{\widetilde{X}} \cdot F=\pi^{*} K_{X} \cdot F+\frac{1}{n+1} V \cdot F=0+\frac{1}{n+1} \cdot \frac{1}{n} \cdot(-n-1)=-\frac{1}{n} .
$$

Note that $F$ and $V$ meet the threefold singularity (of type $Q(n-1)$ ) normally at the vertex of $V \cong \overline{\mathbb{F}}_{n}$. If $F$ passes through $E \cap \widetilde{C}$, then $\widehat{F}$ and $\widehat{V}$ also meet the singular point on $\widehat{X}$ normally. If $F \cap E \cap \widetilde{C}=\varnothing$, then $\widehat{X}$ is smooth near $\widehat{F}$. Let $D$ be the generator passing through $E \cap \widetilde{C}$, as shown in Figure 5 . 

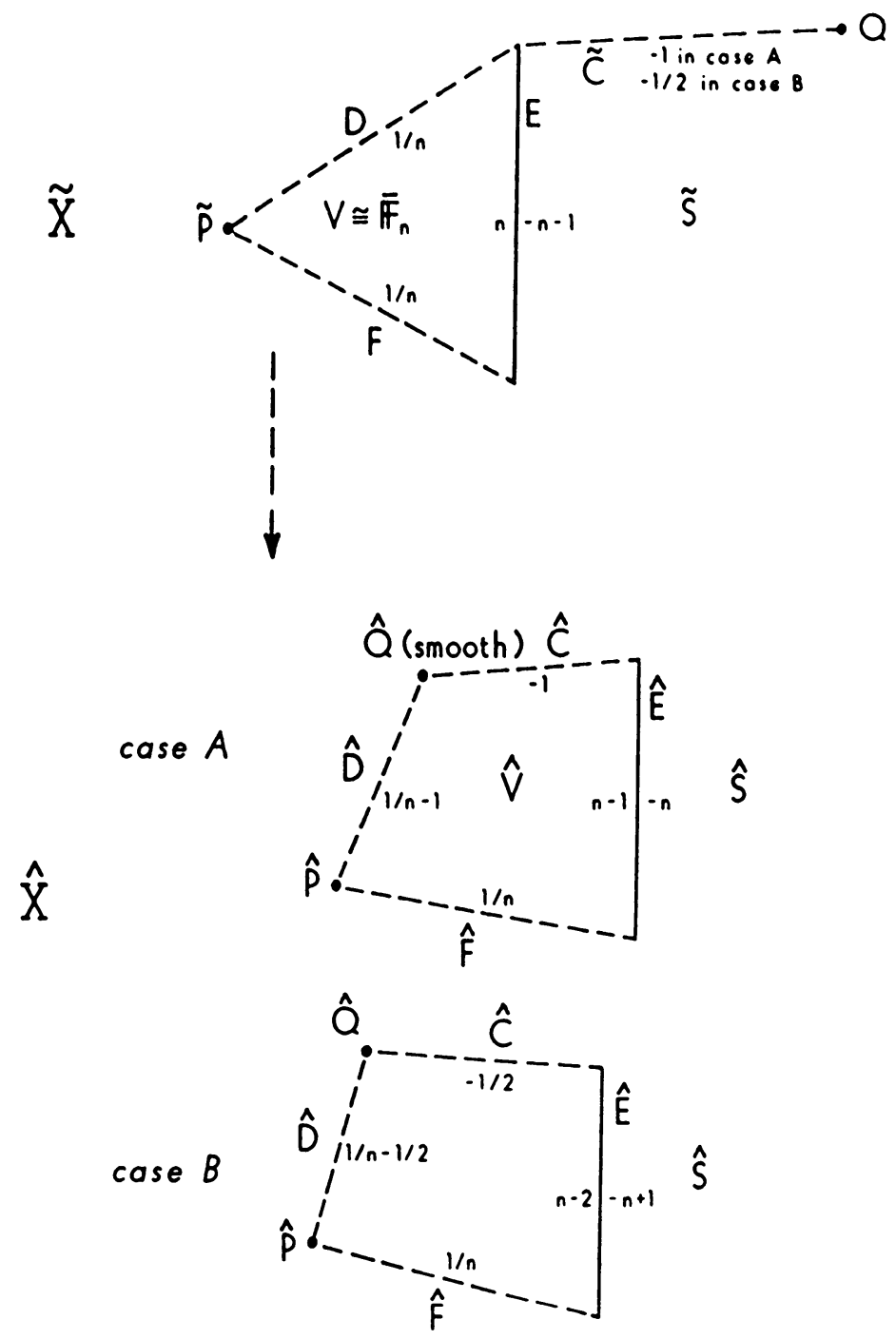

FIGURE 5. $\tilde{X} \rightarrow \widehat{X}$

We begin the induction in case A with $n=1$. Then $V \cong \mathbb{P}^{2}$ and $\widehat{V} \cong \mathbb{F}_{1}$, since $\widetilde{X} \rightarrow \widehat{X}$ blows up $\widetilde{C}$, which meets $V$ normally. Now $E \cdot V=\left(E^{2}\right)_{\widetilde{S}}=$ -2 , so since $\tilde{X} \rightarrow \widehat{X}$ also blows down $\widetilde{C}$, an exceptional curve on $\widetilde{S}, \widehat{E} \cdot \widehat{V}=$ $\left(\widehat{E}^{2}\right)_{\widehat{S}}=\left(E^{2}\right)_{S}+1=-1$, so $\widehat{V}$ may be smoothly contracted along its ruling by a morphism $\widehat{X} \rightarrow \bar{X}$ to a smooth curve in a smooth threefold, i.e. with only "singularities" of type $Q(0)$.

The induction in case B begins with $n=2$, which implies that $K_{\widetilde{X}} \cdot F=-\frac{1}{2}$. For $F$ a generator on $V$ not passing through $E \cap \widetilde{C}, \widehat{F}$ is the total transform of $F$ in $\widehat{X}$, so $K_{\widehat{X}} \cdot \widehat{F}=-\frac{1}{2}$. Again, since $n=2$, on $V, F \equiv \frac{1}{2} E$, so $\widehat{F} \equiv \frac{1}{2} \widehat{E}$ on $\widehat{V}$ (since $F \cap E \cap \widetilde{C}=\varnothing$ ) and thus $K_{\widehat{X}} \cdot \widehat{E}=-1$. Now $\widehat{E}$ is a smooth rational curve in $\widehat{X}$, which is smooth near $\widehat{E}$, so by adjunction, since 
$K_{\widehat{X}} \cdot \widehat{E}=-1, \operatorname{det} N_{\widehat{E} / \widehat{X}}=-1$. Thus, since $\widehat{E}=\widehat{V} \cap \widehat{S}$ and $\widehat{V}$ and $\widehat{S}$ meet normally, $\left(\widehat{E}^{2}\right)_{\widehat{V}}+\left(\widehat{E}^{2}\right)_{\widehat{S}}=-1$.

Since $n=2,\left(E^{2}\right)_{\widetilde{S}}=-3$. If $\tau$ is the morphism $\widetilde{S} \rightarrow \widehat{S}$, then $\tau^{*} \widehat{E}=E+\widetilde{C}$. By passing to the resolution of $\widetilde{S}(\widetilde{S}$ has an ordinary double point at $Q \in \widetilde{C})$, it is easy to see that $\left(\widehat{E}^{2}\right)_{\widehat{S}}=\left(\left(\tau^{*} \widehat{E}\right)^{2}\right)_{\widetilde{S}}=-1$. Thus $\left.N_{\widehat{S} / \widehat{X}}\right|_{\widehat{E}}=\widehat{E} \cdot \widehat{V}=\left(\widehat{E}^{2}\right)_{\widehat{S}}=-1$ and, by the formula above, $\left(\widehat{E}^{2}\right)_{\widehat{V}}=0$. Therefore $\widehat{V}$ is ruled with fiber $\widehat{E}$ and is generically contractible to $\widehat{C}$. Now all fibers of $\widehat{V}$ are irreducible and all smooth except for the proper transform of the fiber through $E \cap \widetilde{C}$ which has a $Q(1)$-singularity coming from the vertex of $V$ and an $A(m)$-singularity coming from the modification $\widetilde{X} \rightarrow \widehat{X}$. Thus $\widehat{V}$ satisfies the conditions of Corollary 4.5 , so there is a morphism $\widehat{X} \rightarrow \bar{X}$ where $\bar{X}$ is smooth (type $Q(0)$ ) except for a single singular point of type $A(m+1)$.

To prove the inductive step in case $\mathrm{A}$, assume that there is a birational modification when $P$ is of type $Q(n-1)$.

Assume $P$ is type $Q(n)$. Let $D$ be the generator on $V$ through $E \cap \widetilde{C}$ and $F$ any other generator on $V$, so under $\widetilde{X} \rightarrow \widehat{X}$, the total transform of $F$ is $\widehat{F}$, while that of $D$ is $\widehat{D}+\widehat{C}$; moreover, since on $V, D \equiv F$, on $\widehat{V}, \widehat{F} \equiv \widehat{D}+\widehat{C}$. Now $K_{\widetilde{X}} \cdot F=K_{\widetilde{X}} \cdot D=-\frac{1}{n}$, so $K_{\widehat{X}} \cdot \widehat{F}=-\frac{1}{n}$ and $K_{\widehat{X}} \cdot \widehat{D}=-\frac{1}{n}-K_{\widehat{X}} \cdot C$. Since $N_{\widehat{C} / \widehat{X}} \cong \mathscr{O}_{\widehat{C}}(-1) \oplus \mathscr{O}_{\widehat{C}}(-1), K_{\widehat{X}} \cdot \widehat{C}=0$ and so $K_{\widehat{X}} \cdot \widehat{D}=-\frac{1}{(n-1)+1}$. On the other hand, since $D$ is a fiber on an $\overline{\mathbb{F}}_{n},\left(D^{2}\right)_{\widehat{V}}=\frac{1}{n}$, so $\left(\widehat{D}^{2}\right)_{\widehat{V}}=\frac{1}{n}-1=-\frac{(n-1)}{(n-1)+1}$. Thus $\widehat{D} \subset \widehat{V} \subset \widehat{X}$ satisfies the inductive hypotheses, with $\widehat{D}$ containing a singular point of type $Q(n-1)$. Letting $\widehat{X} \rightarrow \overline{\bar{X}}$ be the birational modification centered at $\widehat{C}$, then by $4.6 .3, \overline{\bar{V}}$ is $\widehat{V}$ with $\widehat{D}$ contracted to a $Q(n-2)$ point on $\overline{\bar{X}}$ and $\overline{\bar{V}} \cong \overline{\mathbb{F}}_{n-1}$ (we have performed an elementary modification in the sense of ruled surfaces on $\widetilde{V}$ ). By 3.1.3, $\overline{\bar{V}}$ may now be contracted by a morphism $\overline{\bar{X}} \rightarrow \bar{X}$ yielding a singularity of type $Q(n-1)$.

Finally, to prove the inductive step in case $B$, assume that there is a birational modification when one singular point $P$ is of type $Q(n-1)$. Assume $P$ is of type $Q(n)$. Letting $D$ be the generator on $V$ through $E \cap \widetilde{C}$ and $F$ any other generator on $V$, so as in the induction step for case A, $K_{\widehat{X}} \cdot \widehat{F}=-\frac{1}{n}$ and $K_{\widehat{X}} \cdot \widehat{D}=-\frac{1}{n}-K_{\widehat{X}} \cdot \widehat{C}$. By Proposition 4.3.4, $K_{\widehat{X}} \cdot \widehat{C}=0$ also, so again $K_{\widehat{X}} \cdot \widehat{D}=$ $-\frac{1}{n}=-\frac{1}{(n-1)+1}$ and $\left(D^{2}\right)_{\widetilde{V}}=\frac{1}{n}$. On $\widehat{V}, \widehat{F} \equiv \widehat{D}+\widehat{C},\left(\widehat{F}^{2}\right)_{\widehat{V}}=\frac{1}{n},\left(\widehat{C}^{2}\right)_{\widehat{V}}=-\frac{1}{2}$ by 4.3.4, and $\widehat{D} \cap \widehat{C}$ is an ordinary point on $\widehat{V}$, so $\left(\widehat{D}^{2}\right)_{\widehat{V}}=\frac{1}{n}-\frac{1}{2}=\frac{1}{2}-\frac{(n-1)}{(n-1)+1}$, so again $\widehat{D} \subset \widehat{V} \subset \widehat{X}$ satisfies the inductive hypotheses. Thus there is $\widehat{X} \rightarrow \overline{\bar{X}}$ with $\overline{\bar{V}} \equiv \mathbb{F}_{n-3}$ and a singular point of type $A(m+1)$ off $\overline{\bar{V}}$. Contracting $\overline{\bar{V}}$ by $\overline{\bar{X}} \rightarrow \bar{X}$ yields the second singularity, of type $Q(n-2)$, as required.

\section{Proof of the Main Theorem}

In this final section, we prove our Main Theorem 2.7 in the case in which $Y_{\min } \neq X$. Recall from 2.8 that cases (i), (ii) and (iii) of the Main Theorem are those for which $Y_{\min }=X$ and follow directly from the canonical multiplicities of flower components from Table 6 . Similarly we saw that $Y_{\min } \neq X$ precisely 
when $Y$ has "bad" flowers: those of types $4 B, 6 B, 6 D, 6 \beta, 8 \beta$ or $12 \beta$. In the presence of such flowers, we must contract both $\Theta$, the proper transform of the pot in $X$, and the "residual canonical" flowers, i.e. those components of flowers or partially contracted flowers with the same canonical multiplicity as that of $\Theta$. These residual canonical flowers are those of types $3 B, 4 \alpha, 6 \alpha$, $6 E, 8 \alpha$ and $12 \alpha$ as well as the bottom component from $4 C$ flowers and the bottom two components from $6 C$ flowers (see Table 6). The proof proceeds as follows: in 5.1, we compute the normal bundle and canonical divisor (as $\mathbb{Q}$-Cartier divisor) of $\Theta$ and consider the "logarithmic version" of the theory of extremal rays and minimal models to the pair $(\Theta, \Delta)$, where $\Delta=-N_{\Theta / X}$. If $K_{\boldsymbol{\Theta}}+\Delta$ is nef, then we show that $K_{X} \equiv 0$, while if $K_{\boldsymbol{\Theta}}+\Delta$ is not nef, then we apply Mori theory to the pair in Lemma 5.2. These results ensure us of the following: first (5.2.1), that if the pair has an extremal curve with negative self-intersection, then it is the type of curve we can flip using the flips of Proposition 4.6; second (5.2.2 and 5.2.3) that when there are no more extremal curves of negative self-intersection for the pair, then $\Theta$ is contractible, either as a ruled surface or a surface of Picard number one; and, finally, that the possible types and combination of types of bad and residual canonical flowers is severely limited (5.2.2 and 5.2.3). The results of 5.2 allow us in 5.3 to complete the proof of our Main Theorem 2.7 by flipping until there are no more extremal curves for the pair $(\Theta, \Delta)$, then contracting $\Theta$, contracting the remaining residual canonical flowers to get $Y_{\min }$ and then computing that $K_{Y_{\min }} \equiv 0$ as claimed.

5.1. The pot $\Theta$ and singularities of $X$. Let $\Theta$ be the image of the pot on the model $X$. We can write an appropriate $\mathbb{Q}$-multiple of the fiber as $\Theta+\sum \alpha_{i} \Theta_{i}$. (Here, the $\alpha_{i}$ are positive rational numbers.) Then the normal bundle of $\Theta$ on $X$ takes the form $N_{\Theta / X}=-\sum \alpha_{i} C_{i}$, where $C_{i}=\Theta_{i} \cap \Theta$. If we restrict $K_{X}$ to $\boldsymbol{\Theta}$, we get $\left.K_{x}\right|_{\boldsymbol{\Theta}} \equiv K_{\boldsymbol{\Theta}}+\sum \alpha_{i} C_{i}$. Let $\Delta=\sum \alpha_{i} C_{i}$ (a $\mathbb{Q}$-divisor on $\boldsymbol{\Theta}$ ). The divisor $\Delta$ can be written as a sum of several parts, corresponding to the different types of residual flowers. Namely, we write

$$
\Delta=\Delta_{\text {canon }}+\Delta_{\text {bad }}
$$

where

$$
\Delta_{\text {canon }}=\frac{1}{3} \Delta_{3 B}+\frac{1}{2} \Delta_{4 \alpha C}+\frac{2}{3} \Delta_{6 \alpha C E}+\frac{3}{4} \Delta_{8 \alpha}+\frac{5}{6} \Delta_{12 \alpha}
$$

and

$$
\Delta_{\text {bad }}=\frac{1}{4} \Delta_{4 B}+\frac{1}{3} \Delta_{6 \beta D}+\frac{1}{6} \Delta_{6 B}+\frac{1}{4} \Delta_{8 \beta}+\frac{1}{6} \Delta_{12 \beta},
$$

where the subscripts refer to the types of flowers.

The singularities on $\Theta$ and $X$ are related as shown on Table 7 , where a singularity of type $2 B C$, for example, refers to the singularity on $\Theta$ from the

TABLE 7. Surface versus threefold singularities

\begin{tabular}{|c|c|}
\hline Singularities in $\Theta$ & Singularities in $X$ \\
\hline $4 D$ & $P(n)$ \\
$2 B C$ & $A(m)$ \\
$4 A$ & $Q(3)$ \\
$3 A, 4 A^{\prime}$ & $Q(2)$ \\
$2 A, 3 A^{\prime}, 4 A^{\prime \prime}$ & $Q(1)$ \\
\hline
\end{tabular}


contraction of the $2 B$ or $2 C$ flower, and so on. Primes, double primes and so on refer to images after flips, since, as we shall see in 5.2 and 5.3, if an exceptional curve of negative self-intersection passes through a point of type $3 A$ (or $4 A$ ), then there will prove to be a flip in the threefold of the form described in Proposition 4.6 $\mathrm{A}$ and so by 4.6 .3 the $n$ of the $Q(n)$ will be lowered by one, although the surface singularity will be unchanged. Note that $3 A^{\prime \prime}$ and $4 A^{\prime \prime \prime}$ are smooth points on $X$.

We apply the "logarithmic version" of the theory of extremal rays to the pair $(\Theta, \Delta)$. That is, we want to find a series of extremal curves to contract which will either make the image of $K_{\Theta}+\Delta$ into a nef divisor, or allow the image of $\Theta$ to be contracted on the image of $X$. If $K_{\Theta}+\Delta$ is nef, then $\left.K_{X}\right|_{\Theta} \equiv 0$ (see the definition of $\Delta$ above). By definition of $\alpha_{i}$ 's, we have $\Theta+\sum \alpha_{i} \Theta_{i} \equiv X_{0} \equiv 0$ and $\Delta=-\left.\Theta\right|_{\Theta}=\left.\sum \alpha_{i} \Theta_{i}\right|_{\Theta}$. Let $K_{X} \equiv r \Theta+\sum r_{i} \Theta_{i}$ for some rational numbers $r$, $r_{i}$. Restricting this expression for $K_{X}$ to $\Theta$, we see that $\left.\sum\left(-r \alpha+\alpha_{i}\right) \boldsymbol{\Theta}_{i}\right|_{\Theta} \equiv 0$ and so $r_{i}=r \alpha_{i}$ for all $i$. Thus $K_{X} \equiv r\left(\Theta+\sum \alpha_{i} \Theta_{i}\right) \equiv 0$. If, on the other hand, $K_{\Theta}+\Delta$ is not nef, then the following lemma is a straightforward (albeit tedious) exercise.

5.2. Lemma. Let $(\Theta, \Delta)$ be a pair arising from the image of a pot on the model $X$, or from the image of such a pair under a sequence of contractions of logextremal curves. Suppose that $K_{\boldsymbol{\Theta}}+\Delta$ is not nef. Then either

(i) $(\Theta, \Delta)$ has an extremal curve $C$, of one of the following types:

(i.1) $C$ meets $\Delta_{4 B}$, passes through one point of type $4 A, 4 A^{\prime}$, or $4 A^{\prime \prime}$, and passes through at most one point of type $2 B C$.

(i.2) $C$ meets $\Delta_{6 \beta D}$, passes through one point of type $3 \mathrm{~A}$ or $3 \mathrm{~A}^{\prime}$, and passes through at most one point of type $2 B C$.

(i.3) $C$ meets $\Delta_{4 B}$ with multiplicity two, passes through one point of type $2 A$, and passes through at most one point of type $2 B C$.

(i.4) $C$ meets $\Delta_{3 B}$ and $\Delta_{6 B}$, and passes through one point of type $2 A$ (In this case, $C$ cannot pass through a point of type $2 B C$.) or

(ii) $\Theta$ is a ruled surface over a curve $\Gamma$ which has at worst ordinary double points, such that each fiber of the ruling is either a smooth rational curve disjoint from the singular locus, or a smooth rational curve counted with multiplicity two which passes through two of the ordinary double points. The generic fiber $\mathscr{F}$ of the ruling is an extremal curve for $(\Theta, \Delta)$. Moreover, the generic fiber $\mathscr{F}$ and the base $\Gamma$ satisfy one of the following (where it is assumed that all types of component of $\Delta$ which are not mentioned are in fact empty):

(ii.1) $\mathscr{F} \cdot \Delta_{4 B}=4$, and $\Gamma$ is rational.

(ii.2) $\mathscr{F} \cdot \Delta_{6 \beta D}=3$, and $\Gamma$ is rational or elliptic. If $\Gamma$ is elliptic, $\boldsymbol{\Theta}$ must be nonsingular.

(ii.3) $\mathscr{F} \cdot \Delta_{6 B}=2, \mathscr{F} \cdot \Delta_{6 \alpha C E}=1$, and $\Gamma$ is rational. $\Theta$ must be nonsingular. (In fact, $\Theta \cong \overline{\mathbb{F}}_{6}$, and $\Delta_{6 \alpha C E}=\sigma_{\infty}$ is the curve with negative self-intersection.)

(ii.4) $\mathscr{F} \cdot \Delta_{8 \beta}=2, \mathscr{F} \cdot \Delta_{4 \alpha C}=1$, and $\Gamma$ is rational or elliptic. $\Theta$ must be nonsingular. (In fact, if $\Gamma$ is rational, then $\Theta \cong \overline{\mathbb{F}}_{4}$, and $\Delta_{4 \alpha C}=\sigma_{\infty}$ is the curve with negative self-intersection.)

(ii.5) $\mathscr{F} \cdot \Delta_{12 \beta}=1, \mathscr{F} \cdot \Delta_{6 \beta D}=1, \mathscr{F} \cdot \Delta_{4 \alpha C}=1$, and $\Gamma$ is elliptic. $\Theta$ must be nonsingular, or

(iii) $\Theta$ is a surface with Picard number one, any curve on $\Theta$ is extremal 
for $(\Theta, \Delta)$, and exactly one type of component of $\Delta$ is nonempty. (In fact, the nonempty type must be one of: $\Delta_{4 B}, \Delta_{6 \beta D}, \Delta_{6 B}, \Delta_{8 \beta}$, or $\Delta_{12 \beta}$. Moreover, if the nonempty type is $\Delta_{8 \beta}$, then $\Theta \cong \mathbb{P}^{2}$, while if it is $\Delta_{12 \beta}$, then $\Theta \cong \overline{\mathbb{F}}_{3}$.)

Remarks. (1) There is a bit of overlap between cases (i) and (ii): the extremal curves in case (i) may contract to a point, or may be $\frac{1}{2}$ of a fiber of a ruling on $\Theta$. This latter occurs in (i.1) when the singularities are of types $4 A^{\prime \prime}$ and $2 B C$; in (i.2) when the singularities are of types $3 A^{\prime}$ and $2 B C$; and in (i.3) when the singularities are of types $2 A$ and $2 B C$. (The corresponding rulings are of types (ii.1), (ii.2), and (ii.1), respectively.)

(2) The cases leading to Kodaira fibers (as discussed in Theorem (4.2) of [CM1]) are (ii.2), (ii.4), and (ii.5), when $\Gamma$ is an elliptic curve. (The Kodaira fibers are of types IV, III, and II, respectively.) These cases also correspond to Ueno's cases 14, 21, and 19, respectively. (See $\S 1$.)

(3) Ueno's case 4 gives a $\Theta$ which, after blow-downs of type (i.3), falls in case (ii.1); Ueno's case 10 gives a $\Theta$ which, after blow-downs of type (i.4), falls in case (iii) (with $\Delta_{6 B}$ nonempty).

5.3. The final sequence of flips and contractions. Here we will bring together the results of $\S \S 4,5.1$ and 5.2 to complete the proof of the Main Theorem 2.7 for the remaining case (in which $Y$ has at least one bad flower). In $\S 5.1$ we saw that if $K_{\Theta}+\Delta$ is nef, then $K_{X} \equiv 0$, as desired. Lemma 5.2 shows that if $K_{\boldsymbol{\Theta}}+\Delta$ is not nef, then there exist certain extremal curves on $\Theta$. Assume for the moment that such curves are of the types in 5.2(i), so then (referring to Table 7) the extremal curve $C$ is a smooth rational curve, meeting at most two singular points of $X$, one of type $Q(n), n=1,2$ or 3 , and possibly another of type $A(m)$, depending on whether $C$ passes through a point of type 2BC on $\Theta$. Computing $\left(C^{2}\right)_{\Theta}$ shows that the conditions of Proposition 4.6 have been met and so there is a flip centered at $C$. Let $\bar{X}$ be the resulting threefold and $\bar{\Theta}$ the resulting surface. Since $X \rightarrow \bar{X}$ is an isomorphism off $C$ and $\bar{C}$ and $C$ is not a double curve of $X_{0}$, all the notations and result of 5.1 and 5.2 remain valid with a bar on top of each symbol with the same singularities as in Table 7. We will therefore use $X, \Theta$ and so on to refer to what is, properly speaking, $\bar{X}, \bar{\Theta}$ and so on. Thus we can continue performing these flips until either $K_{\Theta}+\Delta$ is nef, in which case the theorem is proved, since then $K_{X} \equiv 0$, or there are no more extremal curves of the type in 5.2(i).

Assume that $\Theta$ has an extremal curve of the type in 5.2(ii), so $\Theta$ is a ruled surface. By $4.5, \Theta$ can be contracted in $X$ to produce a new threefold $X^{\prime}$. Now if $X$ only has one type of bad flower and no canonical flowers, then it is easy to see that $K_{X^{\prime}} \equiv 0$ and so $X^{\prime}$ is our $Y_{\min }$. This is precisely the case in 5.2(ii.1) and 5.2(ii.2). In 5.2(ii.3), we have residual canonical flowers of types $6 B$ and $6 \alpha C E$ meeting a nonsingular ruled pot-image $\Theta$. The curve of intersection between $\Theta$ and the set of $6 \alpha C E$-flowers is a single section on $\Theta$, so in particular there is exactly one $6 \alpha C E$-flower. After $\Theta$ is contracted along its ruling, the bottom component of the residual $6 \alpha C E$-flower remains nonsingular near the image of $\Theta$, since the curve of intersection was a section. The components of that flower can now be successively contracted, from the bottom up. (As Table 6 shows, there are 1 or 2 components in the flower.) The result is a model of $X$ with numerically trivial canonical divisor: $Y_{\min }$. In case 5.2(ii.4) we have residual flowers of types $8 \beta$ and $4 \alpha C$ meeting a 
nonsingular ruled pot-image $\Theta$. The curve of intersection between $\Theta$ and the set of $4 \alpha C$-flowers is a single section on $\Theta$, so in particular there is exactly one $4 \alpha C$-flower. After $\Theta$ is contracted along its ruling, the residual $6 \alpha C E$-flower remains nonsingular near the image of $\Theta$, since the curve of intersection was a section. That flower can now be contracted; the result is $Y_{\min }$. Finally, in case 5.2(ii.5) we have residual flowers of types $12 \beta, 6 \beta D$, and $4 \alpha C$ meeting a nonsingular ruled pot-image $\Theta$. The curve of intersection between $\Theta$ and the set of $6 \beta D$-flowers is a single section on $\Theta$, so in particular there is exactly one $6 \beta D$-flower. Similarly, there is exactly one $4 \alpha C$ flower. After $\Theta$ is contracted along its ruling, both the residual $6 \beta D$-flower and the residual $4 \alpha C$-flower remain nonsingular near the image of $\Theta$, since the curves of intersection were sections. The $4 \alpha C$ flower can now be contracted, after which the $6 \beta D$-flower can be contracted. The result is a model of $X$ with numerically trivial canonical divisor.

Our final case is that of 5.2(iii), in which $\Theta$ has Picard number one. Since $\Delta$ is a nonzero effective divisor of $\Theta, \Delta$ is ample and so $N_{\Theta / X}=-\Delta$ is antiample. Thus $\Theta$ is contractible. Since exactly one type of component of $\Delta$ is nonempty, it is easy to see that the canonical bundle is numerically trivial after $\Theta$ is contracted, completing the proof of Main Theorem 2.7.

\section{APPENDIX 1. A CORRECTION FOR UENO'S PAPER [U2]}

Two of the cases in Ueno's second paper [U2] contain errors which we would like to correct. In brief, Ueno missed some fixed points for the group action in each case, and therefore missed some components of the central fiber in the minimal desingularization.

The cases in question are case $12^{1}$ and case $13^{2}$, treated on pp. 172-177 and 178-182 of [U2], respectively. Some of the results about these cases appear in a table on p. 166 of [U2]; a corrected version of the relevant lines of the table appears as our Table 8. Our Figures 6 and 7 provide corrected illustrations of the central fibers, which should replace the illustrations on pp. 167 and 168 of [U2], respectively.

Ueno's construction in each of the cases proceeds as follows. (We follow his notation as closely as possible.) Let $E$ be a disk in $\mathbb{C}$, let $E_{z}$ denote the elliptic curve $\mathbb{C} /(\mathbb{Z}+\mathbb{Z} z)$, and let $\rho=e^{2 \pi i / 3}$ so that $-\rho^{2}=e^{2 \pi i / 6}$. We fix a complex number $z$, and let $\mathscr{B}$ be the family of abelian surfaces $E \times E_{\rho} \times$ $E_{z}$, with coordinates $\left(\sigma,\left[\zeta_{1}, \zeta_{2}\right]\right)$. (The square brackets indicate equivalence $\bmod (\mathbb{Z} \oplus \mathbb{Z} \rho, \mathbb{Z} \oplus \mathbb{Z} z).) \mathscr{B}$ is acted on by a cyclic group $G$ of order 6 , generated by

$$
g:\left(\sigma,\left[\zeta_{1}, \zeta_{2}\right]\right) \rightarrow\left(e^{2 \pi i / 6} \sigma,\left[-\rho^{k} \zeta_{1},-\zeta_{2}\right]\right)
$$

where $k=1$ in case 12 , and $k=-1$ in case 13 . The degeneration in question is a desingularization of $\mathscr{B} / G$.

The construction of the desingularization begins by considering $\mathscr{B}_{1}=E \times E_{\rho}$. The action of $g^{2}$ fixes the third factor $E_{z}$ and induces an automorphism $h$ of $\mathscr{B}_{1}$ which generates a cyclic subgroup $H$ of order 3 . Explicitly, $h$ is given by

$$
h:\left(\sigma,\left[\zeta_{1}\right]\right) \rightarrow\left(\rho \sigma,\left[\rho^{-k} \zeta_{1}\right]\right),
$$

\footnotetext{
${ }^{1}$ Case 12 corresponds to the conjugacy class III (1)(a) on p. 47 of [U1].

${ }^{2}$ Case 13 corresponds to the conjugacy class III (1)(b) on p. 47 of [U1].
} 


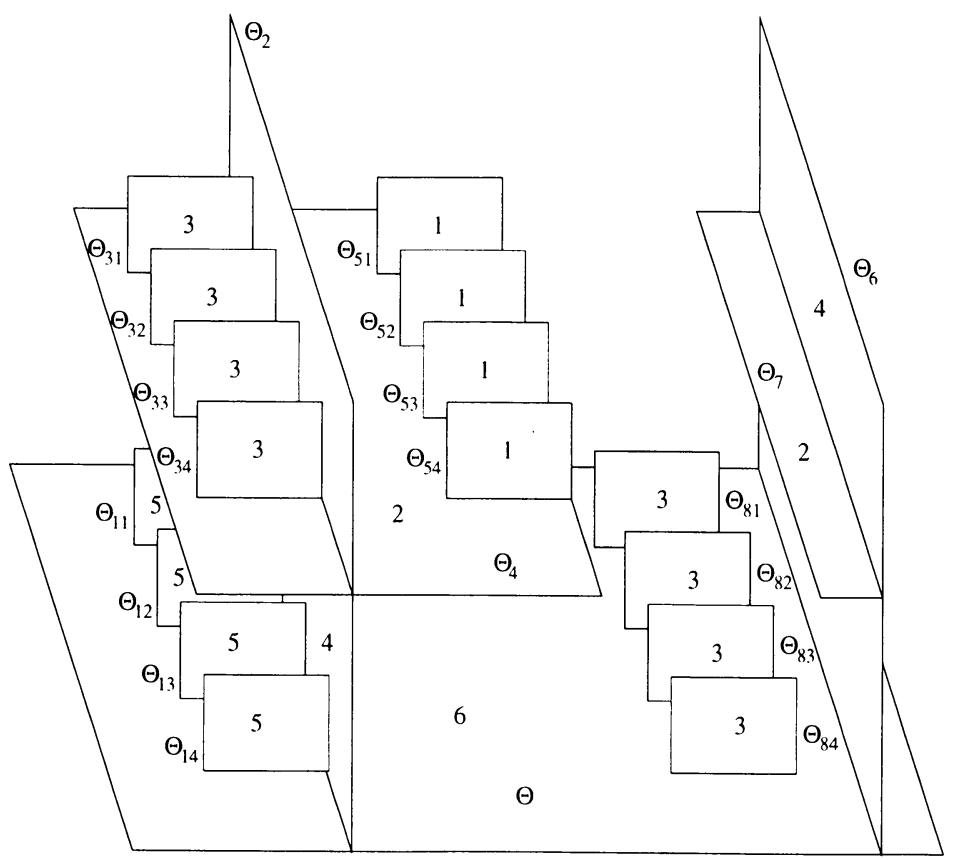

FIGURE 6

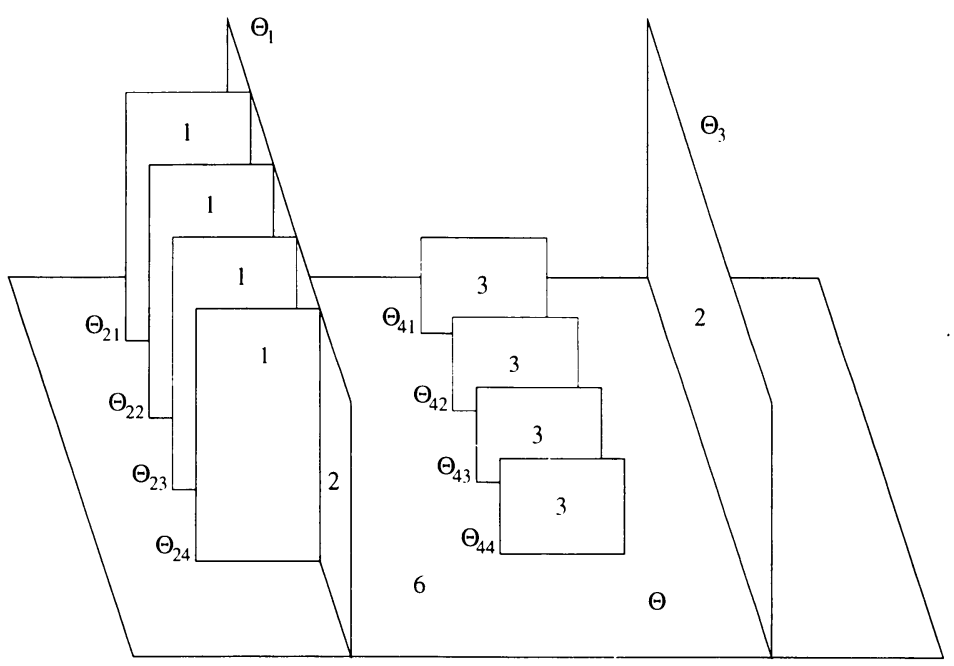

FIGURE 7

TABLE 8

\begin{tabular}{|c|c|c|}
\hline III & (1) (a) & $6 \boldsymbol{\Theta}+5 \sum_{j=1}^{4} \boldsymbol{\Theta}_{1_{j}}+4 \Theta_{2}+3 \sum_{j=1}^{4} \Theta_{3_{j}}+2 \Theta_{4}+\sum_{j=1}^{4} \Theta_{S_{j}}+4 \Theta_{6}+2 \Theta_{7}+3 \sum_{j=1}^{4} \Theta_{8_{j}}$ \\
\hline & (b) & $6 \Theta+2 \Theta_{1}+\sum_{j=1}^{4} \Theta_{2_{j}}+2 \Theta_{3}+3 \sum_{j=1}^{4} \Theta_{4_{j}}$ \\
\hline
\end{tabular}


since $\rho^{2 k}=\rho^{-k}$. Ueno first constructs a desingularization $\mathscr{A}_{1} \rightarrow \mathscr{B}_{1} / H$. (This is just the minimal normal crossings model for the elliptic surface with Kodaira fiber of type $\mathrm{IV}^{*}$, resp. type IV, in cases 12 , resp. 13.) $g$ then lifts to an automorphism $\tilde{g}$ of $\mathscr{A}_{1} \times E_{z}$ (of order 2), and the final family $\mathscr{A}$ is a desingularization of the quotient $\left(\mathscr{A}_{1} \times E_{z}\right) / \widetilde{G}$.

Unfortunately, Ueno missed 4 of the fixed points of $\tilde{g}$ acting on $\mathscr{A}_{1} \times E_{z}$. To see these additional points, we introduce some new coordinate charts (in which they become more easily visible) to be added to the ones used by Ueno. Let $b_{\mu}, \mu=1,2$ or 3 , denote the nontrivial points of order 2 on $E_{\rho}$, numbered so that the automorphism $\left[\zeta_{1}\right] \rightarrow\left[\rho \zeta_{1}\right]$ induces the permutation (123) on the indices. (So for example, we may choose $b_{1}=\frac{1}{2}, b_{2}=\frac{\rho}{2}, b_{3}=\frac{1+\rho}{2}$.) The new coordinates to introduce on $E_{\rho}$ are $w_{\mu}^{1}=\zeta_{1}-b_{\mu}$. Notice that the automorphism $\left[\zeta_{1}\right] \rightarrow\left[\rho^{-k} \zeta_{1}\right]$ maps the coordinate $w_{\mu}^{1}$ to the coordinate $w_{\mu-k}^{1}$ (where $\mu-k$ is computed $\bmod 3$ ), while the automorphism $\left[\zeta_{1}\right] \rightarrow\left[-\rho^{k} \zeta_{1}\right]$ maps the coordinate $w_{\mu}^{1}$ to the coordinate $-w_{\mu+k}^{1}$ (computing $\left.\mu+k \bmod 3\right)$.

The pair $\left(\sigma, w_{\mu}^{1}\right)$ gives local coordinates on $\mathscr{B}_{1}$ with center $\left(0, b_{\mu}\right)$. The automorphism $h$ maps $\left(\sigma, w_{\mu}^{1}\right)$ to $\left(\rho \sigma, w_{\mu-k}^{1}\right)$ (where $\mu-k$ is computed $\bmod 3)$. Thus, in $\mathscr{B}_{1} / H$, any of the coordinates $\left(\sigma, w_{\mu}^{1}\right)$ will serve as local coordinates on a neighborhood of the common image point $(0, b)$ (where $b \equiv$ $\left.b_{\mu} \bmod H\right)$, with the identifications

$$
\left(\sigma, w_{\mu}^{1}\right) \sim\left(\rho \sigma, w_{\mu-k}^{1}\right) \sim\left(\rho^{2} \sigma, w_{\mu+k}^{1}\right) .
$$

These same functions serve as coordinates on $\mathscr{A}_{1}$, since $\mathscr{A}_{1}$ is isomorphic to $\mathscr{B}_{1} / H$ in a neighborhood of $(0, b)$.

The automorphism $\tilde{g}$ of $\mathscr{A}_{1} \times E_{z}$ can be expressed in these coordinates as

$$
\left(\sigma, w_{\mu}^{1},\left[\zeta_{2}\right]\right) \rightarrow\left(-\rho^{2} \sigma,-w_{\mu+k}^{1},\left[-\zeta_{2}\right]\right) \sim\left(-\sigma,-w_{\mu}^{1},\left[-\zeta_{2}\right]\right) .
$$

It follows that there are four isolated fixed points for the action of $\tilde{g}$ mapping to $(0, b)$ in $\mathscr{B}_{1} / H$, namely the points $(0,0,0),\left(0,0, \frac{1}{2}\right),\left(0,0, \frac{z}{2}\right)$, $\left(0,0, \frac{1+z}{2}\right)$ in the present coordinate system. (These are the four points which Ueno missed.)

The singular locus of the quotient space $\left(\mathscr{A}_{1} \times E_{z}\right) / \widetilde{G}$ thus has four components which were omitted by Ueno-one point $P_{j}$ for each of these fixed points. These quotient singularities are each of type $N_{1,1}^{2}$ in Ueno's notation, and of type $Q(1)$ in the notation of this paper.

The exceptional divisor in Ueno's "canonical resolution" of these singularities in a $\mathbb{P}^{2}$; this desingularization agrees with the one we use in this paper. We thus get four new components of the central fiber, each isomorphic to $\mathbb{P}^{2}$. We have named these components $\Theta_{8 j}$ in case 12, and $\Theta_{4 j}$ in case 13. (Here, $j$ ranges between 1 and 4.) These components appear in Table 8 by these names, and appear in Figures 6 and 7 as the components labeled with 3's which meet the bottom component $\Theta$ (labeled with a 6) but no other component.

To complete our analysis, we must verify that the components $\Theta_{8 j}$ (resp. $\Theta_{4 j}$ ) do in fact have multiplicity 3 in the fiber, as the table and figures assert. To do this, we must compute the function $(\sigma)^{6}$ in coordinates on the desingularization, and discover the multiplicity of the exceptional divisor in the divisor of that function. Following Ueno's notation, a typical coordinate chart $\widetilde{V}_{1}$ of 
the desingularization is as follows. (See pp. 56-57 of [U1].) The coordinates are $\left(\tilde{v}_{1}^{1}, \tilde{v}_{1}^{2}, \tilde{v}_{1}^{3}\right)$, and the desingularization of $P_{j}$ is defined by the meromorphic map

$$
T_{V_{1}}:\left(\sigma, w_{1 i}, \zeta_{2}\right) \mapsto\left(\sigma / w_{\mu}^{1},\left(w_{\mu}^{1}\right)^{2}, \zeta_{2} / w_{\mu}^{1}\right)
$$

(that is, $\left.\tilde{v}_{1}^{1}=\sigma / w_{\mu}^{1}, \tilde{v}_{1}^{2}=\left(w_{\mu}^{1}\right)^{2}, \tilde{v}_{1}^{3}=\zeta^{2} / w_{1 i}\right)$. It follows that $(\sigma)^{6}=$ $\left(\tilde{v}_{1}^{1}\right)^{6}\left(\tilde{v}_{1}^{2}\right)^{3}$. In this chart, the exceptional divisor here is defined by $\tilde{v}_{1}^{2}=0$, while the proper transform of $\Theta$ is defined by $\tilde{v}_{1}^{1}=0$; thus, the exceptional divisor has multiplicity 3 in the fiber.

\section{APPENDIX 2. ERRATA FOR [CM1]}

We would like to correct a few typographical errors from [CM1], on which the second part of this paper is based. First, on p. 359, the phrase in Definition (3.1) should be: let $\Sigma_{i}=F_{i-1} \cap F_{i}$. Second, in the middle of p. 362, the formula for the genus of $D$ should read $g(D)=1+k^{2} M / N$. Third, Table 3.4 on $\mathrm{p}$. 367 should be repaced by the version given below. And finally, the first formula on p. 368 should read

$$
\Delta_{\chi}\left(\mathscr{O}_{X_{t}}\right)=\sum s_{i} \chi\left(\mathscr{O}_{V_{i}}\right)-\frac{1}{2} \sum_{i<j}\left(s_{i}+s_{j}\right) \chi\left(\mathscr{O}_{D_{i j}}\right)-\frac{1}{12} \sum_{i<j}\left[s_{i}-\frac{s_{j}^{2}}{s_{i}}\right]\left(D_{i j}^{2}\right)_{V_{i}}
$$

TABLE 3.4. Flowers with $F_{0} \cong \mathbb{P}^{2}, \Sigma_{1}$ a conic.

\begin{tabular}{|c|c|c|c|c|c|}
\hline & $F$ & $F_{i}, i \geq 1$ & $s\left(F_{l}\right) / s\left(F_{l+1}\right)$ & weight & $\Delta_{\chi}$ \\
\hline $2 B$ & $2 s F_{0}$ & - & 2 & 2 & 0 \\
\hline $2 C$ & $2 s F_{0}+s F_{1}+\cdots+s F_{l}$ & $\mathbb{F}_{2}$ & 1 & 2 & 0 \\
\hline $4 C$ & $2 s F_{0}+s F_{1}+\cdots+s F_{l}$ & $\mathbb{F}_{2}$ & $\frac{1}{2}$ & 4 & 0 \\
\hline $4 D$ & $s F_{0}+\cdots+s F_{l}$ & $\mathbb{F}_{4}$ & 1 & 4 & 0 \\
\hline $6 C$ & $2 s F_{0}+s F_{1}+\cdots+s F_{l-1}+2 s F_{l}$ & Note a & $\frac{2}{3}$ & 6 & $\frac{1}{3} s$ \\
\hline $6 D$ & $2 s F_{0}+s F_{1}+\cdots+s F_{l}$ & $\mathbb{F}_{2}$ & $\frac{1}{3}$ & 6 & $\frac{1}{3} s$ \\
\hline $6 E$ & $2 s F_{0}$ & - & $\frac{2}{3}$ & 6 & $\frac{1}{3} s$ \\
\hline
\end{tabular}

a. In this case, $F_{l} \cong \mathbb{F}_{4}$ and $F_{i} \cong \mathbb{F}_{2}$ for $i<l$.

\section{REFERENCES}

[CM1] B. Crauder and D. R. Morrison, Triple-point-free degenerations of surfaces with Kodaira number zero, The Birational Geometry of Degenerations, Progress in Math. 29, Birkhäuser, Boston, Mass., 1983, pp. 353-386.

[CM2] _ On terminal singularities with triple-point-free resolutions, preprint, $30 \mathrm{pp}$.

[D] V. I. Danilov, The birational geometry of toric 3-folds, Izv. Akad. Nauk SSSR Ser. Mat. 46 (1982), 971-982; English transl. in Math. USSR Izv. 21 (1983)269-280.

[F] A. Fujiki, On resolutions of cyclic quotient singularities, Publ. Res. Inst. Math. Sci. 10 (1974), 293-328.

[G] H. Grauert, Über Modifikationen und exzeptionelle analytische Mengen, Math. Ann. 146 (1962), 331-368.

[II] M.-N. Ishida and N. Iwashita, Canonical cyclic quotient singularities of dimension three, Complex Analytic Singularities, Adv. Studies in Pure Math., no. 8, Kinokuniya, Tokyo and North-Holland, Amsterdam, 1986, pp. 135-151. 
[JM] B. W. Jordan and D. R. Morrison, On the Néron models of abelian surfaces with quaternionic multiplication, J. Reine Angew. Math. (to appear).

[Ka] Y. Kawamata, Crepant blowing-up of 3-dimensional canonical singularities and its application to degenerations of surfaces, Ann. of Math. (2) 127 (1988), 93-163.

[Ko] K. Kodaira, On compact analytic surfaces, II-III, Ann. of Math. (2) 77 (1963), 563-626; 78 (1963) $1-40$.

[Ku] Vik. S. Kulikov, Degenerations of $K 3$ surfaces and Enriques surfaces, Izv. Akad. Nauk SSSR Ser. Mat. 41 (1977), 1008-1042; English transl. in Math. USSR Izv. 11 (1977), 957-989.

[My] M. Miyanishi, Projective degenerations of surfaces according to S. Tsunoda, Algebraic Geometry, Sendai 1985, Adv. Studies in Pure Math. 10, Kinokuniya, Tokyo and North-Holland, Amsterdam, 1987, pp. 415-447.

[Mr] S. Mori, Flip theorem and the existence of minimal models for 3-folds, J. Amer. Math. Soc. 1 (1988), 117-253.

[Mrr1] D. R. Morrison, Semistable degenerations of Enriques' and hyperelliptic surfaces, Duke Math. J. 48 (1981), 197-249.

[Mrr2] _ The birational geometry of surfaces with rational double points, Math. Ann. 271 (1985), 415-438.

[N] A. Néron, Modèles minimaux des variétés abéliennes sur les corps locaux et globaux, Publ. Math. Inst. Hautes Études Sci. 21 (1964), 1-128.

[PP] U. Persson and H. Pinkham, Degenerations of surfaces with trivial canonical bundle, Ann. of Math. (2) 113 (1981), 45-66.

[P] H. C. Pinkham, Factorization of birational maps in dimension 3, Singularities, Part 2, Proc. Sympos Pure Math., vol. 40, Amer. Math. Soc., Providence, RI, 1983, pp. 343-371.

[R1] M. Reid, Minimal models of canonical 3-folds, Algebraic Varieties and Analytic Varieties, Adv. Studies in Pure Math., no. 1, Kinokuniya, Tokyo and North-Holland, Amsterdam, 1983, pp. 131-180.

[R2] _ Decomposition of toric morphisms, Arithmetic and Geometry: Papers Dedicated to I. R. Shafarevich, vol. II: Geometry, Progress in Math., no. 36, Birkhäuser, Boston, Mass., 1983, pp. 395-418.

[R3] _ Young person's guide to canonical singularities, Algebraic Geometry-Bowdoin 1985, Part 1, Proc. Sympos. Pure Math., vol. 46, Amer. Math. Soc., Providence, RI, 1987, pp. 345-414.

[S] V. V. Shokurov, Numerical geometry of algebraic varieties, Proc. Internat. Congr. Math. (Berkeley 1986), vol. 1, Amer. Math. Soc., Providence, RI, 1987, pp. 672-681.

[Tsch] H. Tsuchihashi, Compactification of the moduli spaces of hyperelliptic surfaces, Tôhoku Math. J. 31 (1979), 319-347.

[Tsn] S. Tsunoda, Degenerations of surfaces, Algebraic Geometry, Sendai, 1985, Adv. Studies in Pure Math., no. 10, Kinokuniya, Tokyo and North-Holland, Amsterdam, 1987, pp. 755-764.

[U1] K. Ueno, On fiber spaces of normally polarized abelian varieties of dimension 2, I: Singular fibers of the first kind, J. Fac. Sci. Univ. Tokyo Sect. IA 18 (1971), 37-95.

[U2] _ On fiber spaces of normally polarized abelian varieties of dimension 2, II: Singular fibers of the first kind, J. Fac. Sci. Univ. Tokyo Sect. IA 19 (1972), 163-199. 74078

Department of Mathematics, Oklahoma State University, Stillwater, Oklahoma

Current address: Department of Mathematics, Colorado State University, Fort Collins, Colorado 80523

E-mail address: crauder@math.okstate.edu

Department of Mathematics, Duke University, Durham, North Carolina 27708-0320

E-mail address: drm@math.duke.edu 